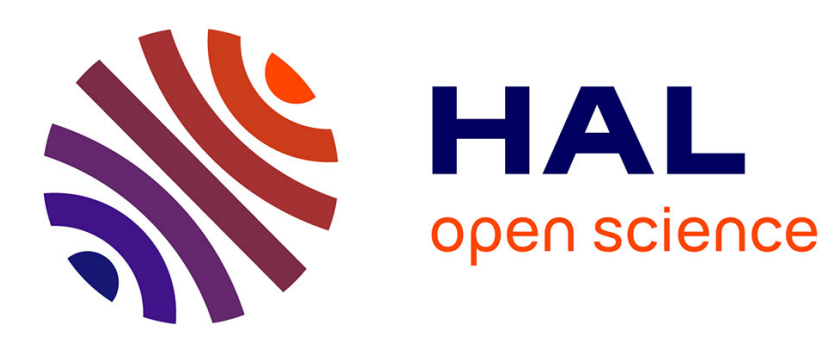

\title{
Lipid bilayer adhesion on sparse DNA carpets: Theoretical analysis of membrane deformations induced by single-end-grafted polymers
}

\author{
F. Thalmann, V. Billot, C. Marques
}

\section{- To cite this version:}

F. Thalmann, V. Billot, C. Marques. Lipid bilayer adhesion on sparse DNA carpets: Theoretical analysis of membrane deformations induced by single-end-grafted polymers. Physical Review E: Statistical, Nonlinear, and Soft Matter Physics, 2011, 83 (6), pp.061922. 10.1103/PhysRevE.83.061922 . hal-03586246

\author{
HAL Id: hal-03586246 \\ https://hal.science/hal-03586246
}

Submitted on 23 Feb 2022

HAL is a multi-disciplinary open access archive for the deposit and dissemination of scientific research documents, whether they are published or not. The documents may come from teaching and research institutions in France or abroad, or from public or private research centers.
L'archive ouverte pluridisciplinaire HAL, est destinée au dépôt et à la diffusion de documents scientifiques de niveau recherche, publiés ou non, émanant des établissements d'enseignement et de recherche français ou étrangers, des laboratoires publics ou privés. 


\title{
Lipid bilayer adhesion on sparse DNA carpets: theoretical analysis of membrane deformations induced by single end-grafted polymers.
}

\author{
F. Thalmann*, V. Billot, and C. M. Marques \\ Institut Charles Sadron, Université de Strasbourg, CNRS UPR 22, \\ 23 rue du Loess, F-67037 Strasbourg Cedex, France
}

(Dated: April 20, 2011)

\begin{abstract}
We consider a single end-grafted polymer chain covered by a membrane in contact with a flat and rigid surface, in the context of supported membrane adhesion on surfaces carrying dilute polymer brushes. The fluid membrane adheres to the surface due to attractive interactions, the presence of a macromolecule locally hinders the membrane-surface contact and creates a protuberant membrane bulge. We study both the size and elevation of such membrane deformations as a function of curvature modulus, surface tension, adhesion energy and chain size. Scaling results are derived, valid for both ideal and nonideal chain statistics, leading to complex diagrams of states depending on curvature modulus, tension and adhesion values. We also compute quantitatively the membrane deformation profile for shallow bulges, and make predictions for realistic systems involving DNA grafted chains covered by lipid membranes.
\end{abstract}

PACS numbers: PACS. 87.16.D-; 82.35.Gh; 87.17.Rt

\section{INTRODUCTION}

Fluid bilayers self-assemble from phospholipid solutions as molecularly thin membranes of roughly $5 \mathrm{~nm}$, building in the living realm the walls of cells and cellular organelles [1]. Phospholipid vesicles and liposomes can also be assembled from aqueous solutions providing simple models to understand cell and cell membrane behavior: adhesion and fusion, mechanical resistance or transport properties. In this context Giant Unilamellar Vesicles or GUV's are of particular interest: they can be conveniently prepared by electroformation, with sizes up to one hundred micrometers and studied by several optical microscopy and micromanipulation methods.

Fluid membranes are often exposed to interactions with other macromolecular species [2-9]. In formulations for detergency, pharmaceutics or cosmetics, polymers are added for performance, processing, conditioning or delivery [10]. A number of formulations based on advanced polymer decorated membranes have been proposed in the emerging context of drug vectorization, encapsulation and targeting [11, 12], interesting examples being for instance those made from a mixture of PEG-lipids and phospholipids [13]. In living organisms the walls of cells and cellular organelles host many large proteins and other biopolymers responsible for the mechanical coupling of the membrane with the cytoskeleton, providing coating protection or engaging in fusion or adhesion events $[14,15]$. The quantitative description of these geometries involving membranes, polymers and interfaces requires considering situations of strong polymer confinement similar to the one that we discuss in this paper.

Understanding the interactions between a polymer chain and a fluid membrane, is thus not only of a clear practical importance, it also poses fascinating questions for the statistical physicist of the soft nanosciences. The physics of simple fluid bilayers and of polymer chains is now well understood. Following seminal work by Helfrich [16] who first recognized the importance of the membrane bending elasticity, and by Edwards and de Gennes who developed the statistical physics tools to deal with chain connectivity, extensive theoretical and experimental studies in polymers and membranes contributed to the writing of one of the finest chapters in modern statistical physics $[17,18]$. But the entropic nature of the interactions between these two classes of soft nanomaterials is still to be understood [19].

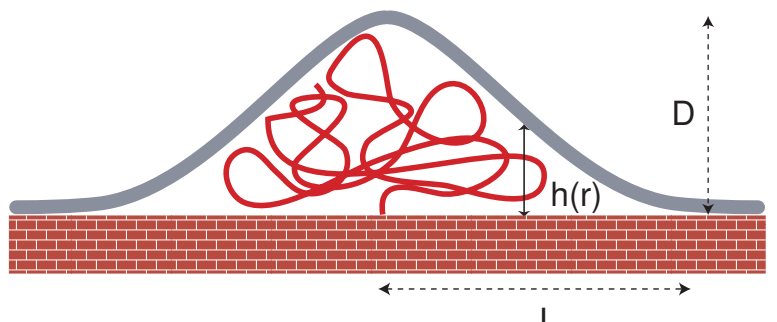

FIG. 1. (Color online) The geometry of polymer-membrane interactions discussed in this paper: a single polymer is endgrafted to a solid substrate and confined between the wall and a fluid membrane. The membrane is homogeneously adhered to the substrate except for the bulge of lateral size $L$ and height $D$ induced by the polymer repulsive forces. These forces result in a deformation profile $h(r)$ that can be exactly computed from polymer theory and the mechanics of fluid membranes.

In the quest of directly measuring the interactions between a fluid bilayer and a single polymer chain, Hisette et. al. [20] recently studied the adhesion of Giant Unilamellar Vesicles on surfaces coated with a carpet of sparsely end-grafted DNAs. The dimensions of the GUVs and of the $\lambda$-phage DNA allowed optical imaging of both 
the membrane deformations and of the DNA average configurations. In a number of cases, spreading of the vesicle adhesion patch, which mostly scrapes and staples the end-grafted DNA chains, also leads to a bulge configuration [21] whereby a DNA chain is confined between the solid substrate and the deformed enveloping fluid bilayer, as sketched in Figure 1. The experiments allow to measure the lateral DNA segment distribution by fluorescence microscopy and the full membrane deformation profile by Reflection Interference Contrast Microscopy, a technique that provides values for the membrane profile with lateral resolution of a fraction of a micrometer and vertical resolution of the order of a few nanometers.

Inspired by the experimental results of Hisette et. al. [20] we study theoretically the polymer confinement and the membrane deformation corresponding to the experimental geometry depicted in Figure (1). The next Section sets the theoretical frame were the polymer membranes interactions will be described: relevant parameters, assumptions and the different physical contributions to the free energy of the system. In Section III we perform a scaling analysis of the different possible confinement states of the polymer and deformation states of the membrane. This allows us to identify the key parameter combinations, expressed as dimensionless numbers, that govern the system behavior and to pictorially describe the relevant regimes as regions of "state-diagrams" built from such numbers. Section IV outlines a self-consistent determination of the membrane shape for confinement under moderate adhesion, most relevant for experiments. Predictions are given for grafted $\lambda$-phage DNAs covered by phospholipid membranes. In the Conclusion we summarize the results presented in this paper. Four technical appendices show respectively how non ideal chain considerations enters into the picture, how to compute the pressure applied by the polymer on the membrane, how to compute the mechanical response and deformation of an adhered membrane to a localized or distributed stress field and how the quantitative approach compares with scaling arguments. A collection of predicted quantitative profiles and bulge sizes is provided as Auxiliary Material [42].

\section{PARAMETERIZATION AND MODEL FREE-ENERGY}

\section{A. Statement of the problem}

We consider a single polymer chain, grafted on a flat, rigid surface and covered by a membrane, while at distance from the chain non-specific adhesive interactions maintain a close contact between the membrane and the surface. Figure 1 illustrates the situation.

Our goal is to predict and describe the relative conformations of the distorted membrane and the squeezed chain underneath. Squeezed chain conformations are relevant to a number of situations, such as dilute polymer brushes under compression, or for the so-called escape transition which predicts the conformational change of a single chain confined by a rigid piston $[22,23]$. In our problem though there is no room for escape, we do not anticipate any sharp conformational change of the chain, but a progressive compaction under a bulge deformation of the membrane, with size inversely related to the confining forces.

For moderate confining forces, the chain remains in a coiled, or mushroom conformation that preserves the rotation invariance of the system. A local elevation of the membrane right above the grafted chain must be observed, that we call bulge, while the membrane remains closely supported by the substrate at distance from the grafting point. The geometric shape of the membrane deformation results from the competition between repulsive forces pushing the membrane away from the substrate and adhesion and mechanical forces keeping the membrane close to the surface. Adhesion drives the maximum possible amount of the membrane into close contact with the substrate, anchoring the membrane and providing a support for the action of membrane forces related to the bending elastic energy and to the membrane tension. The osmotic pressure associated to the confined polymer is the main repulsive force pushing the membrane away from the surface and thus stabilizing the bulge deformation as depicted in Figure 1.

A successful scaling description of bulge conformations requires two characteristic lengths. The first one is the height $D$ measuring the spatial separation between the top of the bulge and the surface. The second one corresponds to the radius $L$ of the circular rim where the membrane comes into contact with the substrate. In addition, one is interested in the total energetic cost $\mathcal{F}$ associated to both membrane deformation and polymer confinement. We argue on general grounds that $D$ cannot exceed the range of the (only) repulsive force, i.e. the gyration radius $R_{g}$ of the end-grafted chain. Based on the ratio $D / L$, we further distinguish shallow bulges characterized by $D / L \ll 1$, from balloon bulges for which $D \sim L$, as depicted in Figure (2b). Balloon bulges may correspond to a combination of strong adhesion, weak curvature modulus and surface tension.

In what follows, we treat the membrane as a nonfluctuating, athermal, mechanical object. Our model membrane has negligible thickness, finite bending and compressibility moduli, and no elastic shear modulus. Such a smectic liquid crystalline state is observed, for instance, in self-assembled amphiphilic lamellar phases, such as unilamellar giant phospholipid vesicles or supported membranes at room temperature. The polymer is treated as a fluctuating chain, grafted by one end on a perfectly flat substrate, with a suitable surface preparation preventing the polymer from adsorbing onto it. The chain is assumed to be flexible and long enough to adopt the so-called mushroom conformation, accurately described by well-established polymer theory. 

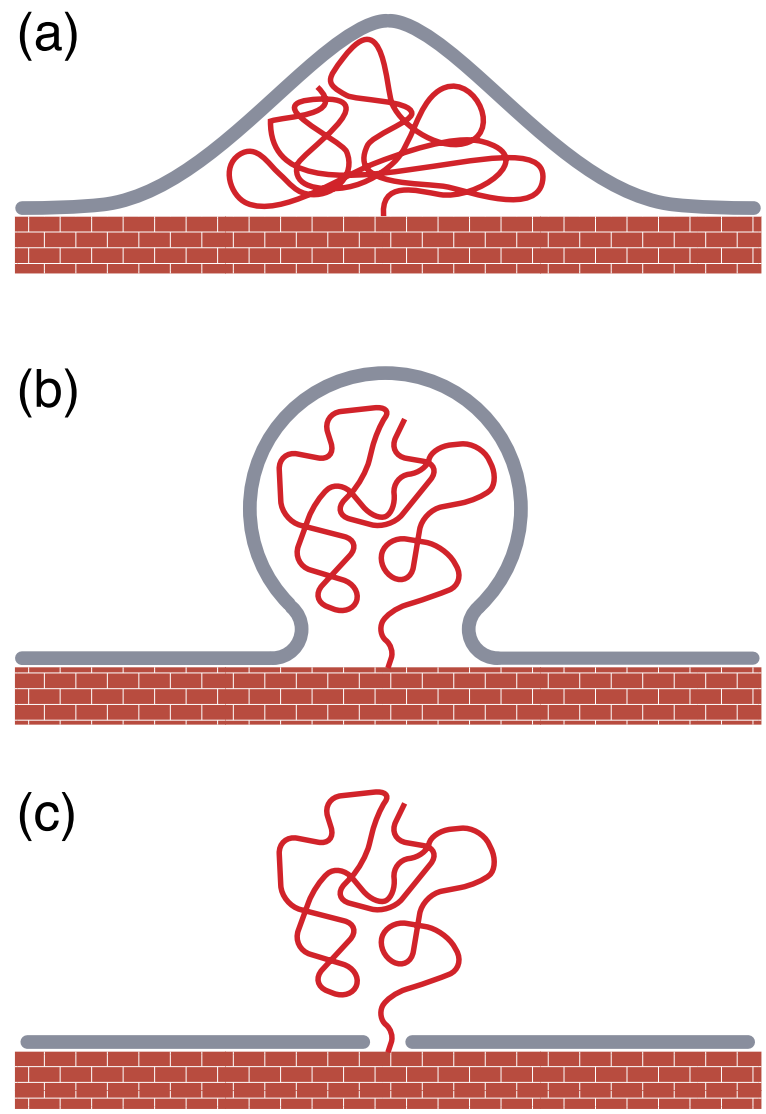

FIG. 2. (Color online) Three possible geometries for a single end-grafted polymer chain confined between a lipid bilayer and the grafting substrate: shallow bulge (a), balloon bulge (b) punctured membrane (c).

\section{B. A configurational free-energy for membrane polymer interactions}

The equilibrium conformation is driven by free-energy minimization. For the geometry described here the free-energy is a sum of four contributions.

\section{Curvature}

The first one is a Helfrich curvature term characterizing the membrane shape deformation. Taking a flat membrane as reference state, the curvature term reads $\mathcal{F}_{\text {curv }}=\kappa \int \mathrm{d} \Sigma \mathcal{C}^{2} / 2$, where $\mathrm{d} \Sigma$ is the surface integration element and $\mathcal{C}$ denotes the mean curvature of the surface. The topological term associated with gaussian curvature does not contribute to energy variations since we consider only membrane deformations with fixed topology. The membrane is flat far from the chain, and it is assumed that $\mathcal{C}$ remains small compared with the inverse membrane thickness, legitimating the quadratic approximation.

\section{Surface tension and adhesion energy}

We group together two closely related terms: a surface free-energy term proportional to the total area $\mathcal{A}=\int \mathrm{d} \Sigma$ of the membrane, and a non specific adhesion contribution proportional to the area of substrate free from any membrane close contact. If one considers a patch of membrane with total area $\mathcal{A}_{0}$, a general expression of the surface related free-energy contributions is given by:

$$
\mathcal{F}_{\text {surf }}=\gamma \Delta \mathcal{A}+w \mathcal{A}^{\prime}
$$

with $\Delta \mathcal{A}$ the area difference between the actual conformation and the reference state, and $\mathcal{A}^{\prime}$ the projected area of the non-adhered part of the membrane onto the substrate, or equivalently the amount of substrate area free from membrane adhesion. This expression can be defined on a more rigorous mathematical footing by defining a typical adhesion length scale threshold $\zeta_{w}$ and introducing an elevation $z$ between membrane and substrate. The surface terms then read:

$$
\mathcal{F}_{\text {surf }}=\gamma \int\left[\mathrm{d} \Sigma-\mathrm{d} \Sigma^{\prime}\right]+w \int \mathrm{d} \Sigma^{\prime} \mathrm{H}\left(z-\zeta_{w}\right),
$$

with $\mathrm{d} \Sigma$ the true surface integration element, $\mathrm{d} \Sigma^{\prime}$ the corresponding projected area onto the substrate, and $\mathrm{H}$ the Heaviside function. $\gamma$ describes the cost of increasing the total area, either by tapping into a surface reservoir, or by stretching the membrane and reducing its surface mass while $w$ is related to the non specific short range interactions of the membrane with the substrate, e.g. accounting for van der Waals attraction, hydration forces, or electrostatic interactions with the supporting material [24]. The parameters $\gamma, \kappa$ and $w$ describe entirely the physical properties of the membrane-surface pair [25].

\section{Chain confinement}

A last term $\mathcal{F}_{\text {chain }}$ accounts for the repulsive force exerted by a confined polymer chain. For ideal chains, its expression is obtained by solving the diffusion (Edwards) equation first, prior to the calculation of the partition function and the related free-energy $[18,26,27]$. Approximated expressions can be obtained, based on scaling arguments, for both ideal and self-avoiding chains, and both cases involve the ratio of a typical confinement length scale $D$ over the gyration radius $R_{g}$ of the chain: $\mathcal{F}_{\text {chain }}=T f_{\text {chain }}\left(D / R_{g}\right)$. Scaling forms are $\mathcal{F}_{\text {chain }} \sim T\left(D / R_{g}\right)^{-2}$ for ideal chains (theta solvent), and $\mathcal{F}_{\text {chain }} \sim T\left(D / R_{g}\right)^{-1 / \nu}$, with Flory exponent $\nu \simeq 3 / 5$, for isotropically confined chains in the presence of excluded volume (good solvent).

The resulting total free-energy reduces to a sum of four integrals running only on the parts of the membrane which are not in close contact with the substrate:

$$
\begin{array}{r}
\mathcal{F}=\gamma \int^{\prime}\left[\mathrm{d} \Sigma-\mathrm{d} \Sigma^{\prime}\right]+w \int^{\prime} \mathrm{d} \Sigma^{\prime}+ \\
\frac{\kappa}{2} \int^{\prime} \mathrm{d} \Sigma \mathcal{C}^{2}+ \\
T f_{\text {chain }}\left(\frac{R_{d}}{D}\right),
\end{array}
$$


where $\int^{\prime}$ runs over the un-adhered part of the membrane, $\mathrm{d} \Sigma$ is the real membrane surface area and $\mathrm{d} \Sigma^{\prime}$ the horizontally projected area. Eq. (3) is the starting point of the present study.

\section{A model with three independent parameters and two natural length scales}

To sum up, the above free energy model depends on four explicit parameters. Two of them, the bending modulus $\kappa$ and the tension $\gamma$ are associated with the membrane. The adhesion parameter $w$ characterizes membrane surface interactions and the effect of the polymer chain is entirely described by the gyration radius $R_{g}$. The relative importance of curvature elasticity with respect to membrane tension is determined by the characteristic length $\xi=\sqrt{\kappa / \gamma}$ : at length scales smaller than $\xi$ curvature elasticity phenomena dominates while tension rules deformations at length scales larger than $\xi$. The membrane shape close to the adhesive surface needs to obey the balance of elastic and adhesive torques, and is therefore governed by the characteristic length scale $\xi_{w}=\sqrt{\kappa / w}[28]$. If one chooses $T$ as the natural energy scale, with Boltzmann constant $k_{B}=1$, and $R_{g}$ as the natural length scale, the three remaining independent parameters $\kappa, \gamma$ and $w$ can be rendered dimensionless by combinations of $T$ and $R_{g}: \bar{\kappa}=\kappa / T, \bar{\gamma}=\gamma R_{g}^{2} / T$ and $\bar{w}=w R_{g}^{2} / T$. The most general solution of such a polymer-membrane-substrate system depends thus on three independent dimensionless parameters $\bar{\kappa}, \bar{\gamma}$ and $\bar{w}$ and on the two length scales $\xi$ and $\xi_{w}$ that emerge from them.

\section{SCALING RESULTS}

\section{A. Scaling approach to shallow bulges}

In establishing the scaling results, it is convenient to pick up $T$ and $R_{g}$ as respectively the energy and length scales. As explained above, all the physical quantities can be expressed with prefactors $T$ or $R_{g}$ and combinations of the three other dimensionless parameters $\bar{\kappa}=\kappa / T$, $\bar{w}=w R_{g}^{2} / T, \bar{\gamma}=\gamma R_{g}^{2} / T$. We search for the scaling behaviors of the shallow bulge - see Figure (2a) - elevation $D$, radius $L$ and free energy $\mathcal{F}$. Using the Monge approximation that holds for small gradient deformations of the membrane and assuming ideal chain scaling for the polymer, estimates of the free-energy contributions are $\gamma \int^{\prime}\left[\mathrm{d} \Sigma-\mathrm{d} \Sigma^{\prime}\right]=\gamma D^{2}, \kappa \int^{\prime} \mathrm{d} \Sigma \mathcal{C}^{2}=\kappa D^{2} / L^{2}$, $w \int^{\prime} \mathrm{d} \Sigma^{\prime}=w L^{2}$ and $\mathcal{F}_{\text {chain }}=T R_{g}^{2} / D^{2}$. The total energy can thus be described as

$$
\mathcal{F}=\kappa\left(\frac{D}{L}\right)^{2}+w L^{2}+\gamma D^{2}+T\left(\frac{R_{g}}{D}\right)^{2}
$$

or in a dimensionless form as

$$
\frac{\mathcal{F}}{T}=\bar{\kappa}\left(\frac{D}{L}\right)^{2}+\bar{w}\left(\frac{L}{R_{g}}\right)^{2}+\bar{\gamma}\left(\frac{D}{R_{g}}\right)^{2}+\left(\frac{R_{g}}{D}\right)^{2},
$$

valid within the two geometric restrictions: $D / L \leq 1$ (shallowness) and $D \leq R_{g}$ (finite range of the repulsive force). Minimizing this expression with respect to $D$ and $L$ gives:

$$
\begin{aligned}
\left(\frac{\kappa}{L^{2}}+\gamma\right) D-\frac{T R_{g}^{2}}{D^{3}} & =0 ; \\
\kappa \frac{D^{2}}{L^{3}}-w L & =0 .
\end{aligned}
$$

\section{Weak surface tension}

In a regime of weak surface tension $(\bar{\gamma} \simeq 0)$ this shape is dominated by the curvature term, and we obtain the following scaling laws:

$$
\begin{aligned}
L & \simeq R_{g} \bar{\kappa}^{1 / 6} \bar{w}^{-1 / 3}, \\
D & \simeq R_{g}(\bar{\kappa} \bar{w})^{-1 / 6}, \\
D / L & \simeq \bar{\kappa}^{-1 / 3} \bar{w}^{1 / 6}, \\
\mathcal{F} & \simeq T(\overline{\kappa w})^{1 / 3},
\end{aligned}
$$

valid while the surface tension $\gamma$ remains lower than a typical value:

$$
\bar{\gamma} \leq \bar{\kappa}^{2 / 3} \bar{w}^{2 / 3}
$$

and consistent with the requirement $\gamma \leq \kappa / L^{2}$.

\section{Strong surface tension}

In the opposite limit $\gamma \geq \kappa / L^{2}$, it is appropriate to neglect curvature in front of surface tension. The scaling form of the surface tension contribution must incorporate a logarithmic correction factor. This can be checked, for instance, in the exact case of a membrane pulled from its center, corresponding to a logarithmic elevation profile $h(r) \sim \ln (L / r)$. As a result:

$$
\frac{\mathcal{F}}{T}=\left[\bar{w}\left(\frac{L}{R_{g}}\right)^{2}+\bar{\gamma} \frac{D^{2}}{R_{g}^{2} \ln \left(L / r_{0}\right)}+\left(\frac{R_{g}}{D}\right)^{2}\right],
$$

where $r_{0}$ is a characteristic length, that in most cases may be identified to $\xi=\sqrt{\kappa / \gamma}$.

The scaling properties of the bubble are modified:

$$
\begin{aligned}
D & \simeq R_{g} \bar{\gamma}^{-1 / 4}, \\
L & \simeq R_{g} \bar{\gamma}^{1 / 4} \bar{w}^{-1 / 2}, \\
D / L & \simeq \bar{\gamma}^{-1 / 2} \bar{w}^{1 / 2}, \\
\mathcal{F} & \simeq T \bar{\gamma}^{1 / 2},
\end{aligned}
$$

where logarithmic terms have been omitted. It is possible to improve upon these scaling results by introducing a realistic pressure profile around the grafting point of 
the chain, and by computing self-consistently the shape of the deformation; our Section IV presents the details of such a procedure. This allows us to go beyond the scaling arguments, to challenge them and to describe the geometric shape of these bubbles.

Our predictions (6) and (9) were successfully compared with numerical profiles, supporting our scaling assumptions in both curvature and tension dominated regimes (Appendix D). We recall that these results hold for confining shallow bulges i.e. when the bulge thickness is smaller than the chain size $D \leq R_{g}$ and for bulge lateral sizes larger than bulge thickness $D / L \leq 1$.

\section{B. Scaling of balloon bulges}

In the opposite situation where $D \sim L$, we may speculate on the existence of a balloon shaped configuration, with an isotropically confined chain surrounded by a vesicle-like membrane, as depicted in Figure 2(b). The length $L$ is now associated to the "pore" in the confining membrane while a bubble of lateral size $D$ surrounds the chain. In the making of the mushroom conformation, the quadratic curvature energy plays only a role at the contact point between the membrane and the surface where the local curvature is given by $\xi_{w}$. The determination of the final shape is left entirely to the adhesive and surface tension energies, which must balance the osmotic pressure of the chain. In this situation, the surface tension is dominated by the area of the spherical bubble and scales like $\gamma D^{2}$, while the adhesive energy, controlled by the membrane-free surface patch scales like $w L^{2}$. This leads to

$$
\frac{\mathcal{F}}{T}=\bar{\kappa}+\bar{w} \frac{L^{2}}{R_{g}^{2}}+\bar{\gamma} \frac{D^{2}}{R_{g}^{2}}+\frac{R_{g}^{2}}{D^{2}} .
$$

The lateral size $D$ results primarily from the competition between osmotic pressure and surface tension, leading to

$$
\begin{aligned}
D & \simeq R_{g} \bar{\gamma}^{-1 / 4} \\
L & =0
\end{aligned}
$$

The patch size $L$ has to accommodate for the effect of the adhesive energy and for a geometric matching condition with the large bubble. It is undetermined by the present scaling argument, but can be estimated as $L \simeq \xi_{w}$ by recognizing that: i) small length scales are dominated by the curvature modulus rather than by the surface tension and ii) curvature and adhesive torques must balance at the membrane-surface contact point [28]. Assuming such a value for $L$ leads to a total free energy

$$
\frac{\mathcal{F}}{T}=\bar{\kappa}+\bar{\gamma}^{1 / 2}
$$

where curvature and adhesion terms give $\bar{\kappa} T$ and surface tension and chain confinement give $\bar{\gamma} T$.
At the scaling level, mushroom shaped bubbles are dominated by surface tension if $\bar{\gamma}^{1 / 2} \gg \bar{\kappa}$, leading to

$$
\begin{aligned}
D & \simeq R_{g} \bar{\gamma}^{-1 / 4}, \\
L & \simeq R_{g} \bar{\kappa}^{1 / 2} \bar{w}^{-1 / 2}, \\
D / L & \simeq \bar{\gamma}^{-1 / 4} \bar{\kappa}^{-1 / 2} \bar{w}^{1 / 2}, \\
\mathcal{F} & \simeq T \bar{\gamma}^{1 / 2},
\end{aligned}
$$

or are dominated by curvature if $\bar{\gamma}^{1 / 2} \ll \bar{\kappa}$, leading to

$$
\begin{aligned}
D & \simeq R_{g}, \\
L & \simeq R_{g} \bar{\kappa}^{1 / 2} \bar{w}^{-1 / 2}, \\
D / L & \simeq \bar{\kappa}^{-1 / 2} \bar{w}^{1 / 2}, \\
\mathcal{F} & \simeq T \bar{\kappa},
\end{aligned}
$$

while the above analysis is restricted to $D / L \geq 1$, i.e. to balloon bulges.

In the absence of surface tension, curvature alone cannot fix the lateral size $D$ and is unable to compete with the osmotic pressure of the chain. However, the curvature term imposes a continuity condition on the shape of the membrane. Acting together, the geometric matching condition and the adhesive energy control the extension of $L$. If the adhesion energy is strong, one expects the patch size $L$ to get close to its smallest possible value, e.g. the width of the membrane, a few nanometers in the case of phospholipid bilayers.

In order to have a synthetic perspective of the different configurations adopted by our membrane-polymer system as a function of the relative importance of the tension, adhesion and curvature reduced parameters, it is convenient to draw a state-diagram, that we discuss in the following paragraph.

\section{State diagram for end-grafted gaussian polymers confined under a supported bilayer}

We recall that the membrane tension $\gamma$, curvature modulus $\kappa$ and adhesion energy $w$ can be conveniently renormalized by introducing the associated dimensionless quantities $\bar{\kappa}=\kappa / T, \bar{w}=w R_{g}^{2} / T, \bar{\gamma}=\gamma R_{g}^{2} / T$. As we have seen above, for a given set of parameters, there is a preferred conformation minimizing the free-energy, with equilibrium values for the patch radius $L$ and the height $D$. Comparison of the relative values of $D$ and $L$, comparison of $D$ to the chain radius of gyration $R_{g}$ and comparison of the relative importance of tension and curvature energy terms leads to the state diagram presented in Figure (3). We draw the diagram by assuming that the tension is high $\bar{\gamma}>1$, with little loss of generality as we will see below.

The first important feature in the state diagram is the curve of equation $\overline{w \kappa}=\bar{\gamma}^{3 / 2}$, a line of slope -1 in logarithmic coordinates, cutting the $\ln \bar{\kappa}=0$ axis at $\ln \bar{w}=3 / 2 \ln \bar{\gamma}$. This line, shown as line number $\mathrm{I}$ in Figure (3), divides the parameter space into two regions, 


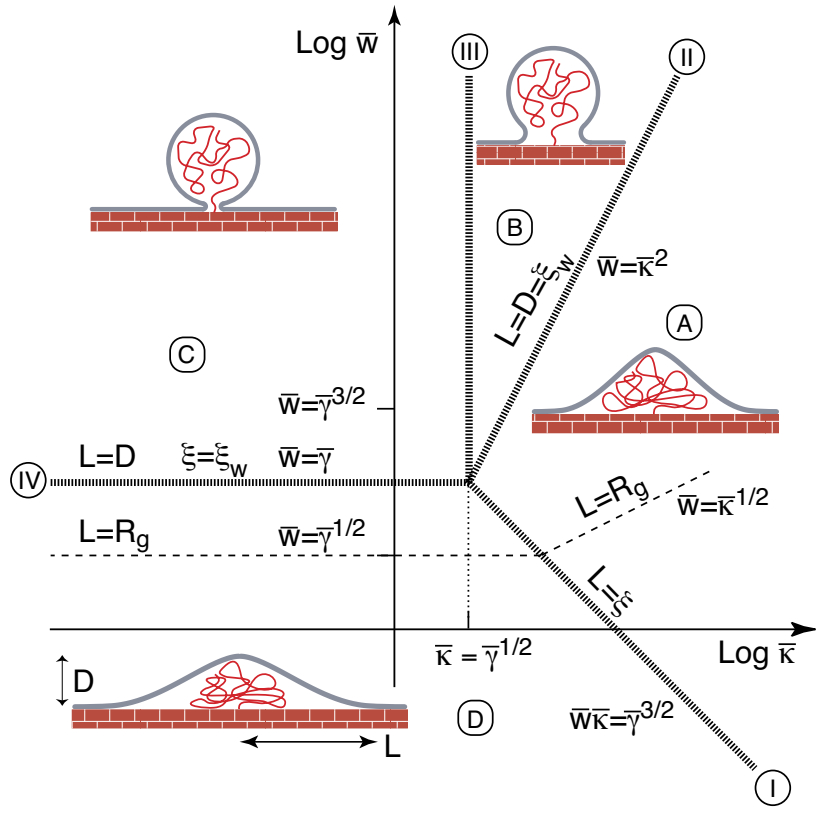

FIG. 3. (Color online) State diagram of the different bulge regimes for an ideal chain - see main text - in logarithmic axes. Horizontal axis is the dimensionless curvature $\bar{\kappa}$, vertical axis is the adhesion parameter $\bar{w}$.

for adhesion strengths $\bar{w}<\bar{\gamma}$. Above the line, the elastic curvature energy dominates, $L<\xi$, while below the line, curvature elasticity is irrelevant and the energy is dominated by membrane tension, $L>\xi$. On the line $L=\xi$.

The second important feature is the curve $\bar{w}=\bar{\kappa}^{2}$ defined by the ratio $D / L$ separating shallow bulges $(D \leq$ $\left.L, \bar{w} \ll \bar{\kappa}^{2}\right)$ and balloon bubbles $\left(L \leq D, \bar{w} \gg \bar{\kappa}^{2}\right)$ in the domain above line I where curvature energy dominates. In the state diagram of Figure (3), this line of slope 2 , shown as line number II where $L=D=\xi_{w}$, intersects the line $\ln \bar{w}=3 / 2 \ln \bar{\gamma}-\ln \bar{\kappa}$ at the point $(\ln \bar{\kappa}=1 / 2 \ln \bar{\gamma}, \ln \bar{w}=\ln \bar{\gamma})$. This is also the point of intersection of the third important curve in the diagram, separating the balloon and the shallow bulge regimes in the domain below line I where membrane tension dominates. This feature is defined by $\bar{w}=\bar{\gamma}$ or equivalently by the horizontal line $(\ln \bar{w}=\ln \bar{\gamma})$, number IV in the diagram.

One can thus identify four regions in the state diagram. For all the four regions, under the assumption that the membrane tension is large enough, $\bar{\gamma}>1$, the polymer chain is always confined, i.e. $D \leq R_{g}$. Region A corresponds thus to confined chains $\left(D \leq R_{g}\right)$ in shallow bulges $(D \leq L)$. Here, the thickness and patch size depend both on curvature elasticity and adhesion strength, but are independent of tension, $D \simeq R_{g}(\overline{\kappa w})^{-1 / 6}$ and $L \simeq R_{g} \bar{\kappa}^{1 / 6} \bar{w}^{-1 / 3}$. Region $\mathrm{B}$ hosts confined chains $\left(D \leq R_{g}\right)$ in balloon bulges $(L \ll D)$. In this region, membrane tension is also negligible, the equilibrium $D$ and $L$ values are independent of $\bar{\gamma}, D \simeq R_{g} \bar{\kappa}^{-1 / 2}$ and
$L=R_{g} \bar{\kappa}^{1 / 2} \bar{w}^{-1 / 2}=\xi_{w}$. By crossing line III into region $\mathrm{C}$, the size of the balloon bulges becomes dependent of membrane tension, $D \simeq R_{g} \bar{\gamma}^{-1 / 4}$, but the size of the patch $L$ is still governed by the usual boundary condition balancing adhesion forces and curvature torque that defines the bulge neck size as $L=\xi_{w}$. This neck starts to open up at $\bar{w}=2 \bar{\gamma}$ close to line IV defined by $\bar{w}=\bar{\gamma}$ that one crosses into region $\mathrm{D}$, a shallow bulge region controlled by membrane tension, where $D \simeq R_{g} \bar{\gamma}^{-1 / 4}$ and the bulge size is given by $L \simeq R_{g} \bar{\gamma}^{1 / 4} \bar{w}^{-1 / 2}$.

Also shown in Figure (3), the dashed lines indicate the crossover from laterally unconfined states of the chain below the lines to laterally confined chains. Note that for the gaussian chain considered here, these lines do not imply any changes in the relative importance of the different terms contributing to the energy. Note also that the minimum value $\bar{\gamma}$ that line IV can assume corresponds to $\bar{\gamma}=1$. For smaller values of $\bar{\gamma}$ the line vanishes from the diagram, with line I fixed for $\bar{\gamma}<1$ at the value $\ln \bar{w}=-\ln \bar{\kappa}$. The conclusions discussed above still hold, with the exception that in regions $\mathrm{C}$ and $\mathrm{D}$, that now merge into a single one, the value of the height $\mathrm{D}$ is kept at the constant value $D \simeq R_{g}$ : the membrane tension in this case $\bar{\gamma}<1$ is not large enough to confine the polymer chain.

For a given value of the membrane tension, the variation of the bulge height $D$ and size $L$ can be displayed either at constant adhesion strength $\bar{w}$ as a function of the elastic modulus $\bar{\kappa}$ or at constant $\bar{\kappa}$ as a function of $\bar{w}$. We chose this last possibility in Figure (4). Given the structure of the state diagram in Figure (3), two cases need to be considered. The first corresponds to weak elastic constants $\bar{\kappa}<\bar{\gamma}^{1 / 2}$, displayed in Figure (4a). The figure shows that the bulge height $D$ is independent of the adhesion strength, and that the patch size $L$ decreases continuously except at $\bar{w} \sim \bar{\gamma}$ where the so called kissing transition $[29,30]$ occurs. There, the neck size decreases from a finite value to $L \sim \xi_{w}$ within a narrow range $\bar{\gamma}<\bar{w}<2 \bar{\gamma}$. For the stronger elastic constants $\bar{\kappa}>\bar{\gamma}^{1 / 2}$, both $D$ and $L$ shown in Figure (4b) decrease continuously as the adhesion strength increases.

\section{Excluded volume and semiflexible chains}

\section{Excluded volume}

The scaling treatment of non-ideal chains, and especially chains swollen by excluded volume leads to a different balance between the several terms contributing to the equilibrium state of the deformed membrane and of the confined polymer chain. This results in a somewhat richer state diagram that the one presented in the previous paragraph for the gaussian chain. We describe these differences in Appendix A where we also present a modified state diagram valid for end-grafted polymer chains in good solvent conditions. 

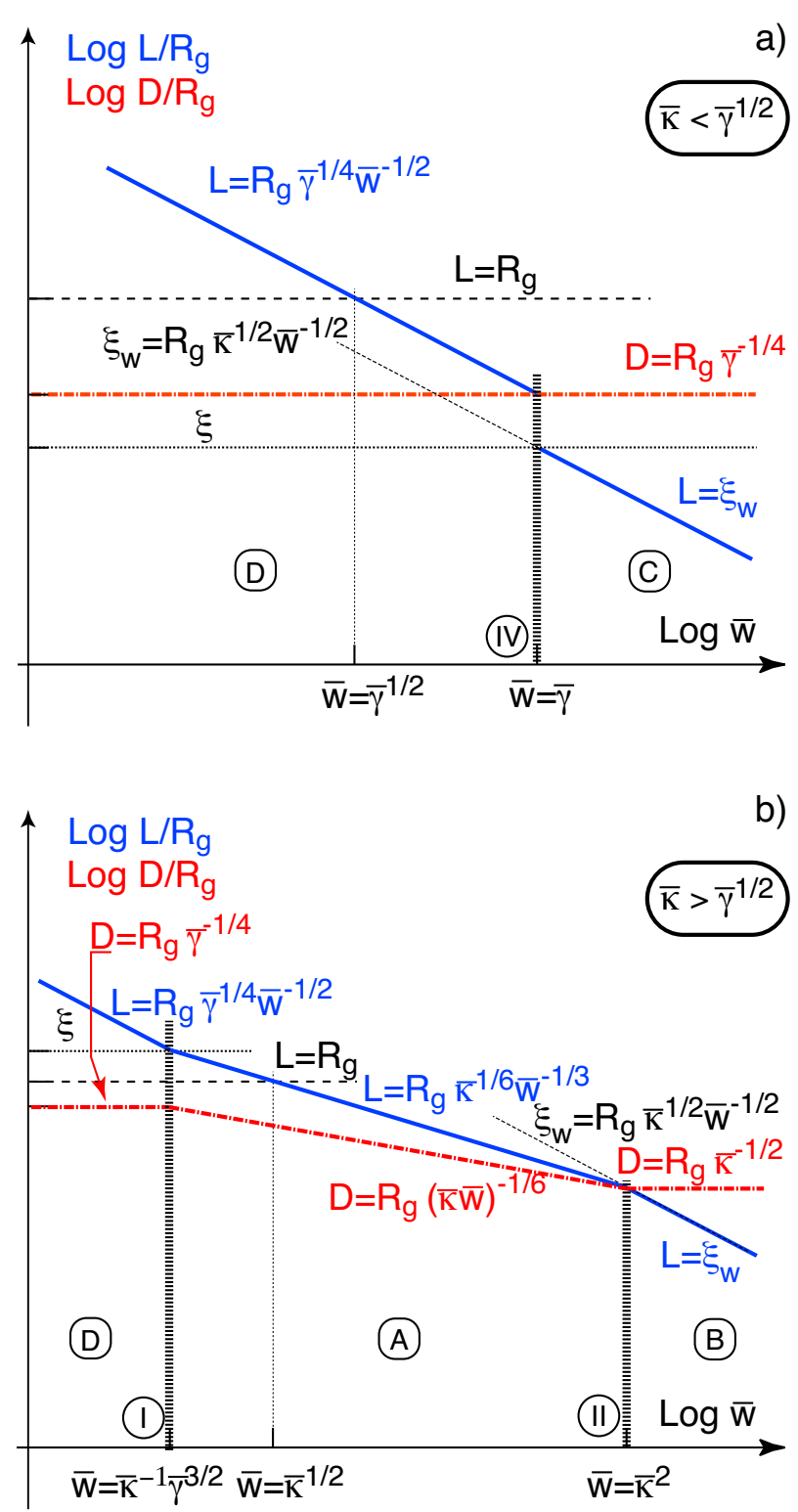

FIG. 4. (Color online) Variation of the bulge height $D$ and size $L$ as a function of the adhesion strength $\bar{w}$ in a logarithmic representation. a) Weak bending rigidities, $\bar{\kappa}<\bar{\gamma}^{1 / 2}$. b) Strong bending rigidities, $\bar{\kappa}>\bar{\gamma}^{1 / 2}$, see also Figure (3)

\section{Semiflexible chains}

Polymers and biopolymers with persistence length $\ell_{p}$ much larger than the monomer size are common. Without interactions between monomers, they behave as gaussian chains on length scales larger than $\ell_{p}$. In three dimensions, excluded volume interactions leading to selfavoiding walk (SAW) behavior only become important for long enough chains. The length scale where chain behavior crosses-over from gaussian to SAW can be estimated from a mean field Flory approach [31-33]. Given a monomer size $a$ that serves as an estimate of the Van der Waals exclusion diameter for the chain, excluded volume interactions become relevant when the dimensionless combination $z=N\left(a / \ell_{p}\right)^{3}$ becomes larger than unity [31]. Therefore, excluded volume corrections in a chain of length $N a$, with $n=N a / \ell_{p}$ persistence lengths, become relevant only for $n \gg\left(\ell_{p} / a\right)^{2}$, and polymer chains of radius $R^{(3 d)}$ is expected to follow gaussian statistics $R^{(3 d)} \sim N^{1 / 2} a\left(\ell_{p} / a\right)^{1 / 2}$ for $\ell_{p} \ll R^{(3 d)} \ll$ $\ell_{p}\left(\ell_{p} / a\right)$ and SAW statistics $R^{(3 d)} \sim N^{3 / 5} a\left(\ell_{p} / a\right)^{1 / 5}$ for $R^{(3 d)} \gg \ell_{p}\left(\ell_{p} / a\right)$.

In two dimensions the effect of excluded volume is much stronger, resulting in full swollen chains for all flexible polymer lengths, the two dimensional chain size being given by $R^{(2 d)} \sim N^{3 / 4} a\left(\ell_{p} / a\right)^{1 / 4}$ for any flexible chain $R^{(2 d)} \gg \ell_{p}$.

A full swollen chain in three dimensions will cross over, as a function of confinement distance $D$ between its three dimensional and its two dimensional radii according to the scaling law $R(D)=R^{(3 d)}\left(R^{(3 d)} / D\right)^{1 / 4}$. Confinement will increase the effect of excluded volume, reducing thus the range over which the chain displays gaussian statistics by a D-dependent factor, $\ell_{p} \ll R^{(3 d)} \ll \ell_{p}(D / a)^{1 / 2}$, a range that vanishes as stated above for full confinement $D \sim a$.

The full analysis of the confinement of a semiflexible chain under an adhered membrane would thus require a combination of the results obtained for gaussian chains with the results presented in the Appendix A, accounting for the possible changes from gaussian to SAW statistics as confinement increases. Such refinements are beyond the scope of this paper where we rather develop in Section IV a self-consistent approach for quantitatively determining the shape of the confining membrane in shallow bulges.

\section{E. Other conformations}

\section{Fully confined polymer conformations}

Purely two-dimensional polymer conformation may result from strong membrane adhesion condition. The chain confinement term $\mathcal{F}_{\text {chain }}$, either reduced to a scaling estimate, or derived in the more formal way of Section IV, cannot account for confinement sizes $D, L$ smaller than a characteristic length $\ell_{p}$, of the size of the persistence length of the chain. In such strong confinement cases, one cannot accurately describe the state of the chain without extra knowledge of its microscopic structure. We adopt here the pragmatic view that situations with $D \leq \ell_{p}$ should be considered as fully confined polymer conformations, without presuming of any actual significant change in the chain behavior.

In the curvature dominated regime, full confinement is achieved by $\overline{\kappa w} \geq\left(R_{g} / \ell_{p}\right)^{6}$. In the tension dominated regime, it is defined by $\bar{\gamma} \geq\left(R_{g} / \ell_{p}\right)^{4}$ 
Similar considerations apply to the membrane thickness $\ell_{m}$ that limit the use of the Helfrich elasticity contribution to deformations obeying $D, L \gg \ell_{m}$.

\section{Anisotropic conformations}

We found no evidence that anisotropic bulge conformations could be thermodynamically favored. Membrane deformations that do not preserve rotational invariance seem disfavored in terms of contact area and confining volume.

However, anisotropic, stretched chain conformations are experimentally observed in the presence of strong, irreversible membrane pinning [20]. Such states can be seen as metastable, dynamically trapped conformations.

\section{Pore formation}

As a last possibility, the chain might punch a pore and diffuse across the membrane, resulting in a flat membrane, and a freely fluctuating grafted chain, see Figure (2c). This case is certainly the most thermodynamically favorable from the point of view of the energy contributions considered so far, as both polymer and membrane maximize their respective free-energy. However, such configuration requires a finite free-energy up to accounting for the opening of the membrane pore that might easily offset the energy gain of releasing both the chain confinement and membrane deformation. Estimations for the pore energy give $E_{p}=2 \pi \Lambda r$, where $r$ is the pore size and $\Lambda=\pi \kappa /\left(2 \ell_{m}\right)$ a line tension that depends on the membrane elasticity $\kappa$ and membrane thickness $\ell_{m}$ [34]. For typical values of fluid bilayers the pore energy for small pores spans the range $5-20 K_{B} T$, comparable to polymer confinement energies. It might be thus possible, by finely controlling the system parameters to prevent or to promote pore formation.

\section{F. Membrane adhesion on decorated substrates}

Studies of lipid bilayers adhesion on substrates serve as model for real biological cell adhesion. An important issue is to understand the adhesion properties of membranes when polymer chains are grafted on the substrate, with the purpose of mimicking real cell environment, such as glycocalix of Gram positive bacteria [15]. Our approach indicates clearly that the global membrane adhesion properties should change significantly when the mutual distance between grafted chains approaches the patch radius $L$. At grafting densities approaching $\sigma_{c} \sim 1 /\left(\pi L^{2}\right)$, one expects the membrane adhesion to be suppressed, or at least strongly reduced. A related discussion can be found in [35] for the formation of surface micelles under confinement membranes.

\section{SELF-CONSISTENT DETERMINATION AND PREDICTION OF SHALLOW BULGES}

\section{A. Prediction of quantitative profiles}

We present in this section a self-consistent numerical determination of the membrane shape. A solution of this kind provides a quantitative prediction of the polymer induced membrane deformation, including numerical prefactors.

Existing quantitative shape determination approaches, based on a Lagrangian mechanics reformulation of the free-energy minimization, have been extensively used for predicting vesicle shapes under constant pressure condition [36] or homogeneous adhesion [37, 38]. The extension of this calculation to the self-consistent, inhomogeneous polymer induced pressure profiles requires matching polymer repulsion and membrane deformation. We present in this section an original and independent numerical scheme that addresses this question, on the basis of the elastic response function of the membrane.

The membrane shape of shallow bulges, characterized by $D \ll L$, deviates gently from the horizontal plane, justifying a Monge approximated estimate of the area increment and the mean curvature. This, in combination with a Derjaguin approximated treatment [39] of the pressure exerted by a confined gaussian chain, enables the quantitative prediction of the shallow bulge deformations. Our results, though restricted to the case of shallow bulges, agree with the scaling results of Section III and may be directly compared with experimental data (Appendix D).

We illustrate our method with the estimation of the deformation of a phospholipid membrane induced by a DNA chain, using some realistic adhesion values suggested by Swain and Andelman [24].

\section{B. Principles of the calculation}

A shallow bulge can be parameterized by a height function $h(r)$ measuring the elevation of the membrane relative to the substrate, at a distance $r$ from the grafting point. Polar coordinates $(r, \theta)$ are used, but for symmetry reasons, the angle $\theta$ does not intervene. Both curvature and surface tension free-energies can be expressed in terms of $h(r)$, especially when gradients of $h(r)$ are small, a necessary condition for shallowness. Taking into account the inhomogeneous pressure term $p(r)$ exerted by the chain, one finds:

$$
\begin{aligned}
\mathcal{F}= & 2 \pi \frac{\kappa}{2} \int_{0}^{L} r \mathrm{~d} r(\Delta h(r))^{2}+2 \pi \frac{\gamma}{2} \int r \mathrm{~d} r(\nabla h(r))^{2} \\
& +w \pi L^{2}-2 \pi \int_{0}^{L} r \mathrm{~d} r p(r ;[h(r)]) h(r) .
\end{aligned}
$$

The pressure term depends on the global shape of the bulge, and, in principle, shows a functional dependence 
on the height profile: $p(r ;[h(r)])$. For a gaussian (ideal) chain, its determination amounts to solving a diffusion problem, with absorbing boundary conditions (the bubble shape) and an initial condition (the grafting point). We use and present in Appendix B an approximated but simpler expression of the pressure field $p(r ; D)$, valid for a constant elevation $D=h(0)$.

Our approach aims at predicting the shape $h(r)$, given the radius $L$ of the bulge, the gyration radius $R_{g}$, the curvature modulus $\kappa$ and the tension $\gamma$. In principle, the numerical value of $L$ should be a prediction and not an input parameter of the model. However, as far as experiments are concerned (say e.g. optical microscopy), the radius $L$ of the bulge can be directly measured, while it is certainly not the case of the adhesion energy $w$, which is "conjugated" to the area $\pi L^{2}$.

The determination of the profile is the outcome of an iterative process. Assuming a numerical input value $D_{i n}$ for the height of the bulge, we compute the pressure term $p\left(r ; D_{i n}\right)$ exerted by a grafted polymer confined between two plates separated by $D_{i n}$. The mechanical equilibrium associated to the above free energy turns out to be:

$$
\kappa \Delta \Delta h(r)-\gamma \Delta h(r)=p\left(r ; D_{i n}\right) .
$$

This relation is then inverted, to get $h(r)$ in terms of $p\left(r ; D_{i n}\right)$, with the help of associated Green functions $G_{\gamma, \kappa, L}\left(r, r^{\prime}\right)$ obeying:

$$
\kappa \Delta \Delta G_{\gamma, \kappa, L}\left(r, r^{\prime}\right)-\gamma \Delta G_{\gamma, \kappa, L}\left(r, r^{\prime}\right)=\frac{\delta\left(r-r^{\prime}\right)}{r} ;
$$

with boundary conditions and analytical expressions that are extensively described in Appendix C, leading to a profile:

$$
h(r)=\int_{0}^{L} r^{\prime} \mathrm{d} r^{\prime} G_{\gamma, \kappa, L}\left(r, r^{\prime}\right) p\left(r^{\prime} ; D_{i n}\right) .
$$

The continuity equation $h(r=0)=D_{\text {out }}$ gives us a fresh estimate of the height of the profile. The numerical scheme $\ldots D_{i} \rightarrow p\left(D_{i}, r\right) \rightarrow h(r) \rightarrow D_{i+1} \ldots$ is then repeated until self-consistence is achieved.

Finally, the validity of the assumption $h(r) \simeq D$ depends on how much $h(r)$ varies in the region where some pressure is exerted. As the range of the pressure field is about $R_{g}$, one must check on our self-consistent solution, that the Derjaguin criterion $\left|h(0)-h\left(R_{g}\right)\right| \ll D$ holds.

\section{Minimization of the free-energy}

In our numerical approach, we decided to work with the radius $L$, rather than with the adhesion parameter $w$. The free-energy $\mathcal{F}\left(T, \kappa, \gamma, L, R_{g}\right)$ to consider is:

$$
\begin{aligned}
\mathcal{F}= & \gamma \pi \int_{0}^{L} r \mathrm{~d} r(\nabla h(r))^{2}+\kappa \pi \int_{0}^{L} r \mathrm{~d} r(\Delta h(r))^{2} \\
& +w \pi L^{2}+\mathcal{F}_{\text {chain }} .
\end{aligned}
$$

Due to the non-vanishing curvature modulus $\kappa$, a second derivative of the height function $h(r)$ exists. As $h(r)$ is continued in the domain $r>L$ by a flat profile $h(r)=0$, we must have for continuity reasons both $h(L)=0$ and $h^{\prime}(L)=0$. A subsequent variation of the functional $\mathcal{F}\left(T, \kappa, \gamma, L, R_{g}\right)$ with respect to $L$ and $h(r)$ and compatible with these boundary conditions gives:

$$
\begin{aligned}
\delta h(L)= & 0 \\
\delta h^{\prime}(L)= & -h^{\prime \prime}(L) \delta L=-\Delta h(L) \delta L \\
\delta \mathcal{F}= & 2 \pi \int_{0}^{L} r \mathrm{~d} r \delta h(r)[-p(r ; h(r))-\gamma \Delta h(r)+ \\
& \kappa \Delta \Delta h(r)]+2 \pi L \delta L\left[w-\frac{\kappa}{2}(\Delta h(L))^{2}\right]
\end{aligned}
$$

We also notice that Laplacian and second derivative at $L$ coincide: $\Delta h(L)=h^{\prime \prime}(L)$. The free-energy variation gives on the one hand the mechanical equilibrium of the membrane $\kappa \Delta \Delta h-\gamma \Delta h=p$, and a mechanical boundary condition $w=\kappa[\Delta h(L)]^{2} / 2$ on the other hand, expressing a balance between the torque associated to the membrane bending and the (non specific) adhesive force.

As a result, this validates our procedure of fixing first $L$ to determine the profile, and then associate an adhesion coefficient parameter $w$ to this profile. In the opposite case of a known $w$, we checked that solving for $L$ the relation $w-\kappa[\Delta h(L)]^{2} / 2=0$ always led to a unique solution, the value of $w$ being rather sensitive to $L$.

Note that advanced concepts of differential geometry and tensor calculus have been recently proposed [40, 41] that enable the precise determination of torques and stresses distributions exerted by membranes of arbitrary conformations. This general formulation reduce to ours in the peculiar quasi-unidimensional model discussed above.

\section{Example of profiles with fixed patch radius $L$ or adhesion $w$.}

If the adhesion parameter $w$ is fixed, and not the patch radius $L$, the numerical value of $L$ itself has to be determined self-consistently. This results in a functional relation $L(w)$ that depends in practice on the other parameters of the problem (see Appendix D). Examples of $L(w)$ and $w(L)$ correspondence are provided (Tables) as Auxiliary Material [42].

We also provide as supplemental material a sample of self-consistent profiles, corresponding to a wide range of values of the curvature modulus $\kappa$, the surface tension $\gamma$ and gyration radius $R_{g}$ [42].

Finally, we show in Appendix D a comparison between our scaling predictions and the outcome of our self-consistent numerical approach. 


\section{E. DNA chains under supported lipid bilayers}

We illustrate the scaling predictions with the case of a single DNA chain grafted on a rigid substrate. Grafting DNA chains on surface is a routine operation, though this may lead to surfaces possessing non trivial adhesion properties. The 48502 base pairs double stranded DNA from $\lambda$ phage virus corresponds to a gyration radius $R_{g} \simeq 0.52 \mu \mathrm{m}$ under standard buffer conditions [20]. This values follows from the commonly accepted values of the persistence length $\lambda_{p}=0.05 \mu \mathrm{m}$ and the DNA contour length $L=16 \mu \mathrm{m}$ [43-45] related to the gyration radius as $R_{g}=\left(\lambda_{p} L / 3\right)^{1 / 2}$. Taking $a=2 \mathrm{~nm}$ as an estimate of a DNA chain diameter, the extension of the gaussian scaling regime ranges between $D=0.05 \mu \mathrm{m}$ and $D=0.3 \mu \mathrm{m}$ (see discussion above), for which $\mathcal{F}_{\text {chain }}=T\left(R_{g} / D\right)^{2}$ is justified. A typical phospholipid membrane curvature modulus in the liquid state is $\kappa=20 k_{B} T$. Swain and Andelman suggest a value of $w=5.1 \times 10^{-6}{\mathrm{~J} . \mathrm{m}^{-2}}$ for a supported membrane in close contact with a smooth surface [24]. The curvature dominated scaling regime predicts:

$$
\begin{aligned}
L & =2.8 \times 10^{-7} \mathrm{~m} \\
D & =1.9 \times 10^{-7} \mathrm{~m} \\
D / L & =0.7 \\
\mathcal{F} & =9 k_{B} T
\end{aligned}
$$

It is indeed difficult to estimate the value of the surface tension $\gamma$ in such an adhesion experiment. The surface tension of giant phospholipid vesicles is usually considered as low, but may increase due to the interaction with the surface. Considering the plausible estimate $\gamma \sim w$, the surface tension dominated regime lead to $L=D=2.3 \times 10^{-7} \mathrm{~m}$ and a ratio $D / L=1$. Moreover, the criterion $\gamma L^{2} \geq \kappa$ is not satisfied, as $\gamma L^{2} / \kappa=0.3$. The scaling approach suggests that the curvature dominated regime is more appropriate to this case.

Observing some membrane deformation with the help of an optical microscope, using for instance a reflection interference contact (RICM) setup [46], ideally requires a spatial extension of order $L \sim 1.5 \mu \mathrm{m}$. This would

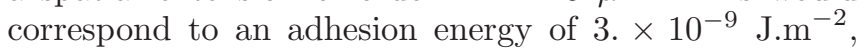
close to the value reported in [24] for the Helfrich regime of supported membrane adhesion.

The scaling predictions for $\kappa=20 k_{B} T, \gamma=w=$ $1.9 \times 10^{-9}{\mathrm{~J} . \mathrm{m}^{-2}}$ and $R_{g}=0.56 \mu \mathrm{m}$ are:

$$
\begin{aligned}
L & =1.8 \times 10^{-6} \mathrm{~m} \\
D & =4.7 \times 10^{-7} \mathrm{~m} \\
D / L & =0.3 \\
\mathcal{F} & =1.4 k_{B} T
\end{aligned}
$$

The ratio $\gamma L^{2} / \kappa \simeq 0.07$ is consistent with a curvature dominated regime.

Our self-consistent quantitative prediction treats on the same footing the curvature and tension dominated regimes. Figure 5 shows the predicted bulge deformation associated with $\kappa=20 k_{B} T, \gamma=w=1.9 \times 10^{-9} \mathrm{~J} . \mathrm{m}^{-2}$ and $R_{g}=0.56 \mu \mathrm{m}$. As the numerical procedure is originally stated in terms of fixed radius $L$, we had to determine first the self-consistent radius $L$ corresponding to

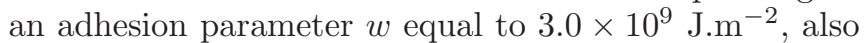
used in our scaling estimate. In the figure, the pressure is represented in arbitrary units and $h(r)$ in micrometers. The maximal elevation $D=h(0)$ reaches $0.5 \mu \mathrm{m}$, while the radius $L$ is about $3 \mu \mathrm{m}$. The ratio $D / L$ justifies the Monge approximation used throughout the numerical procedure while the condition $L \gg R_{g}$ is consistent with our Derjaguin approximation. Such an bulge deformation should fall within reach of optical surface interferometric measurements such as the RICM technique.

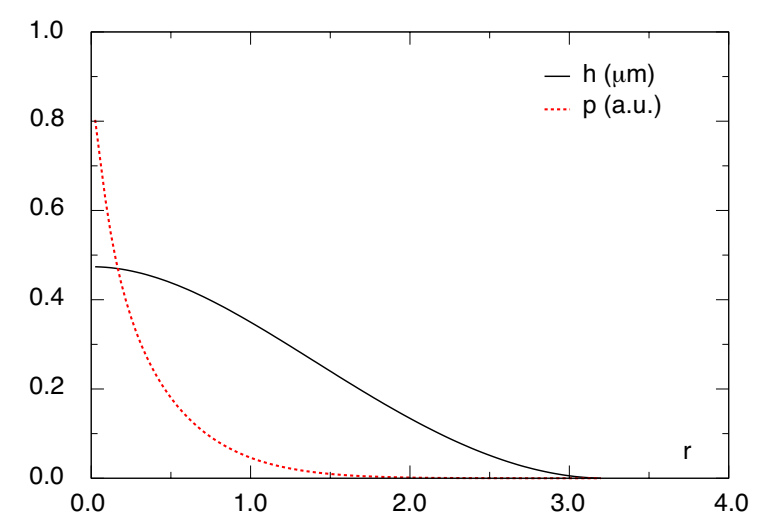

FIG. 5. Membrane deformation profile predicted for $\kappa=$ $20 k_{B} T, \gamma=w=1.9 \times 10^{-9}{\mathrm{~J} . \mathrm{m}^{-2}}^{-2}$ and $R_{g}=0.56 \mu \mathrm{m}$, following values suggested in [24]. The pressure is in arbitrary units.

\section{CONCLUSION}

We considered in this work the case of a single grafted polymer chain surrounded by a membrane adhering onto a flat surface. This situation is relevant for single molecule experiments that are nowadays feasible. This situation is also related to more general problems of membrane non specific adhesion onto decorated substrates.

A scaling description of the interacting membrane, polymer and surface system gave us estimates of the local chain induced deformation, expressed in terms of two characteristic lengths $L$ (size) and $D$ (elevation). Different regimes have been identified and located in a parameter space involving three dimensionless quantities, standing for curvature, tension and adhesion energy. Because we treated the membrane as a classical, non fluctuating object, we did not have to distinguish between local and global surface tension parameters, and dealt with a single quantity $\gamma[47]$. The different regimes that were found are: unconfined chains, confined chains with balloon shapes and confined chains with bulge shape.

Bulge shapes, characterized by a small ratio $D / L$, were further investigated by means of a quantitative and self- 
consistent determination of the elevation profile. Assumptions lying behind this quantitative approach include that the chain must be gaussian (ideal) and that its gyration radius $R_{g}$ must be smaller than $L$ (Derjaguin approximation for the pressure field). A full set of bulge shapes were obtained that depend on three independent dimensionless parameters. We provided a large sample of such bulge shapes and numerical values of $L$ and $D$.

Our approach predicts that a $\lambda$-phage DNA squeezed between a supported membrane and a smooth flat surface should be associated with a suboptical wavelength deformation $L \sim 0.2 \mu \mathrm{m}, D \sim 0.05 \mu \mathrm{m}$, therefore corresponding to a strongly confined chain with bulge deformation. Other adhesion mechanisms, involving specific ligand-receptor bond formation could, however, be associated with larger deformations that could be detected using interference optical microscopy.

An interesting direction for the extension of the present work is to investigate the relaxation dynamics of the condensed membrane-polymer system. For instance, one could envision that a cigar like shape prepared from membrane adhesion spreading could under the appropriate conditions, relax back to a symmetric shape, or that a confined bulge could diffuse laterally if the polymer end attachment was cut.

\section{ACKNOWLEDGMENTS}

The authors thank insightful discussions with A. Schröder.

\section{Appendix A: Non-ideal chains}

\section{Confinement of swollen chains}

One considers a swollen chain in good solvent conditions scaling with Flory exponent $\nu \simeq 3 / 5$, its size being given by the Flory radius $R_{F}=N^{3 / 5} a$. In order to properly account for the confinement of the chain under the different membrane bulges, we first review the chain behavior under confinement inside a disk of radius $\mathrm{L}$ and thickness D and then the confinement inside a sphere of radius $\mathrm{D}$.

Flory chains confined inside a disk of thickness $D$ and radius $L$

- For $0<D<R_{F}$ and $L>R_{F}^{\prime}=R_{F}\left(R_{F} / D\right)^{1 / 4}$, the chain is confined vertically but not laterally, it can be viewed as a $2 \mathrm{~d}$ self-avoiding walk with blobs of size $D$. The scaling form of the confinement energy is similar to the ideal case, albeit with a different exponent:

$$
\mathcal{F}_{\text {chain }}=T\left(\frac{R_{F}}{D}\right)^{1 / \nu}=T\left(\frac{R_{F}}{D}\right)^{5 / 3} .
$$

- For $0<D<R_{F}$ and $L_{0}<L<R_{F}^{\prime}$, with $L_{0}=$ $R_{F}\left(D / R_{F}\right)^{1 / 6}$, the chain is confined both vertically and laterally, it can be viewed as a $2 \mathrm{~d}$ SAW with blobs of size $D$, laterally confined in the disk of size $L$. The lower limit $L_{0}$ denotes a crossover towards a 3d, space-filling conformation of the confinement volume. A scaling expression of the confinement free-energy reads

$$
\mathcal{F}_{\text {chain }}=T\left(\frac{R_{F}}{D}\right)^{5 / 3}\left[1+\left(\frac{L_{0}}{L}\right)^{4}\right] \text {. }
$$

This energy has an extra dependence in $L$, and reduces to a form $\operatorname{Tf}\left(R_{F} / D\right)$ when the lateral confinement contribution is neglected. The magnitude of the $L$ dependent corrective term is small unless $L$ approaches the cross-over characteristic length $L_{0}$. This legitimates the approximation $\mathcal{F}_{\text {chain }} \simeq T\left(R_{F} / D\right)^{5 / 3}$ for $L \gg L_{0}$.

- For $0<D<R_{F}$ and $L<L_{0}$, the scaling picture corresponds to a $3 \mathrm{~d}$ space-filling packing with blobs of intermediate size $\xi_{p}<D$, in the confinement volume $L^{2} D$. In other words, the confined chain behave as a semi-dilute solution of screening length $\xi_{p}$ and energy density $T / \xi_{p}^{3}$. Integrating the energy density over the volume of the chain provides a measure for the energy of confinement of the chain $\mathcal{F}_{\text {chain }}$ :

$$
\begin{aligned}
\xi_{p} & =D\left(\frac{L}{L_{0}}\right)^{3 / 2} \\
\mathcal{F}_{\text {chain }} & =T\left(\frac{R_{F}^{3}}{L^{2} D}\right)^{5 / 4}=T\left(\frac{R_{F}}{D}\right)^{5 / 3}\left(\frac{L_{0}}{L}\right)^{5 / 2}
\end{aligned}
$$

As a summary, for a chain confined inside a disk of radius $L$ and thickness $D$, there are three confinement regimes as a function of the disk size $L$. At large $L$ the chain is only confined vertically and behaves as a two dimensional SAW with $N(a / D)^{5 / 3}$ blobs and blob size $D$. When the disk size reaches the lateral size of the chain $R_{F}^{\prime}=R_{F}\left(R_{F} / D\right)^{1 / 4}$ the chain becomes laterally confined, but the lateral confinement energy is negligible compared to the vertical confinement energy until the chain of blobs has been fully compacted at $L=L_{0}$. For disk radii smaller than $L_{0}$, the disk is homogeneously filled with a semi-dilute solution with a screening length $\xi_{p}$ smaller that the confinement thickness D.

\section{Swollen chains confined inside a sphere of radius $\mathrm{D}$}

Confining isotropically a swollen chain, as it occurs in a balloon shape membrane deformation, brings in blobs 
of intermediate size $0<\xi_{p}<D$.

$$
\begin{aligned}
\xi_{p} & =R_{F}\left(\frac{D}{R_{F}}\right)^{9 / 4} \\
\mathcal{F}_{\text {chain }} & =T\left(\frac{R_{F}}{D}\right)^{15 / 4}
\end{aligned}
$$

The latter expression can be viewed as a special instance of eq. (A4), with $L=D$.

\section{Bulges with excluded volume}

Restricting ourselves first to bulges with $L>L_{0}=$ $R_{F}\left(D / R_{F}\right)^{1 / 6}$ enables us to reiterate the scaling arguments of eq. (6) and eq. (9) by taking instead the expression (A4) for the confinement energy of one chain $\mathcal{F}_{\text {chain }}=T\left(R_{F} / D\right)^{1 / \nu} \simeq T\left(R_{F} / D\right)^{5 / 3}$. This gives:

- for shallow bulges in the regime where curvature dominates,

$$
\begin{aligned}
D & \simeq R_{F} \bar{w}^{-3 / 16} \bar{\kappa}^{-3 / 16} \\
L & \simeq R_{F} \bar{w}^{-11 / 32} \bar{\kappa}^{5 / 32} \\
\mathcal{F} & =T(\overline{w \kappa})^{5 / 16} .
\end{aligned}
$$

- As $L$ decreases and becomes smaller than $L_{0}$, one gets for $D<L<L_{0}=R_{F}\left(D / R_{F}\right)^{1 / 6}$ and by using eq. (A4) instead of eq. (A1)

$$
\begin{aligned}
D & \simeq R_{F} \bar{w}^{1 / 28} \bar{\kappa}^{-9 / 28}, \\
L & \simeq R_{F} \bar{w}^{-13 / 56} \bar{\kappa}^{5 / 56}, \\
\mathcal{F} & =T \bar{w}^{5 / 28} \bar{\kappa}^{15 / 28} .
\end{aligned}
$$

- For shallow bulges dominated by tension, up to logarithmic terms,

$$
\begin{aligned}
& D \simeq R_{F} \bar{\gamma}^{-3 / 11} ; \\
& L \simeq R_{F} \bar{\gamma}^{5 / 22} \bar{w}^{-1 / 2} ; \\
& \mathcal{F}=T \bar{\gamma}^{5 / 11} .
\end{aligned}
$$

- for balloon bulges dominated by surface tension,

$$
\begin{aligned}
& D \simeq R_{F} \bar{\gamma}^{-3 / 11} \\
& L \simeq R_{F} \bar{\kappa}^{1 / 2} \bar{w}^{-1 / 2} \\
& \mathcal{F} \simeq T \bar{\gamma}^{5 / 11} .
\end{aligned}
$$

with ratio $D / L=\bar{\gamma}^{-3 / 11} \bar{\kappa}^{-1 / 2} \bar{w}^{1 / 2}$.

As for the case of gaussian chains, we draw in the following paragraph a state-diagram to summarize the different configurations adopted by our membrane-polymer system as a function of the relative importance of the tension, adhesion and curvature reduced parameters.
3. State diagram for end-grafted swollen polymers confined under a supported bilayer

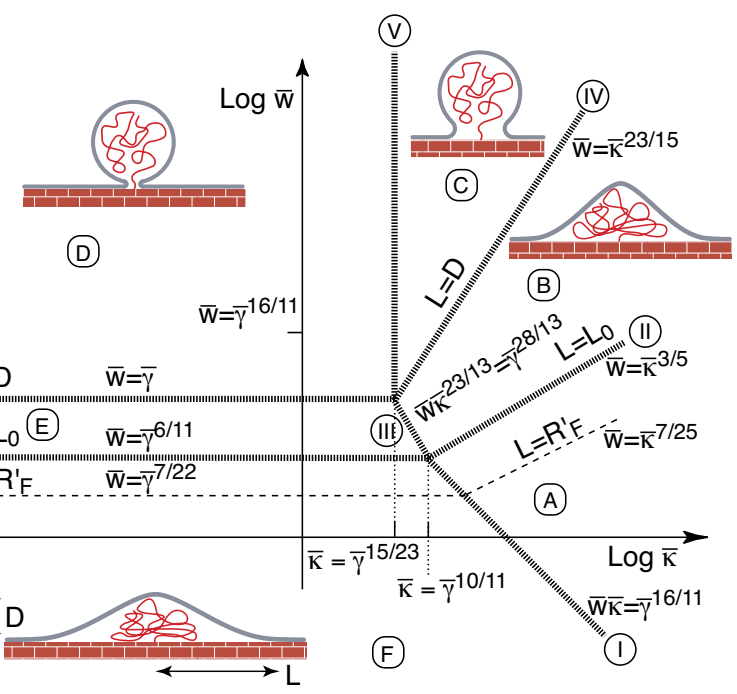

FIG. 6. (Color online) State diagram of the different bulge regimes for swollen chain - see main text - in logarithmic axes. Horizontal axis is the dimensionless curvature $\bar{\kappa}$, vertical axis is the adhesion parameter $\bar{w}$.

The membrane tension $\gamma$, curvature modulus $\kappa$ and adhesion energy $w$, and the associated dimensionless quantities $\bar{\kappa}=\kappa / T, \bar{w}=w R_{F}^{2} / T, \bar{\gamma}=\gamma R_{F}^{2} / T$ constitute, as we have seen above, the set of parameters determining the equilibrium patch radius $L$ and the height $D$ that minimize the free-energy. We adopt a method similar to that of the gaussian chain, and compare the relative values of $D$ and $L$ and the values of $D$ and chain size $R_{F}$ to draw the state diagram presented in Figure (6). We assume as before without loss of generality, that the tension is high $\bar{\gamma}>1$.

The first important feature in the state diagram is the curve of equation $\overline{w \kappa}=\bar{\gamma}^{16 / 11}$, a line of slope -1 in logarithmic coordinates, cutting the $\ln \bar{\kappa}=0$ axis at $\ln \bar{w}=16 / 11 \ln \bar{\gamma}$. This line, shown as line number I in Figure (6), divides the parameter space into two regions, for adhesion strengths $\bar{w}<\bar{\kappa}^{-1} \bar{\gamma}^{16 / 11}$. Above the line, the elastic curvature energy dominates, $L<\xi$, while below the line, curvature elasticity is irrelevant and the energy is dominated by membrane tension, $L>\xi$. On the line $L=\xi$.

The second important feature is the curve $\bar{w}=\bar{\kappa}^{3 / 5}$ defined by $L=L_{0}$, shown as line II in the diagram. Below the line, the confined chain, can be seen as a two dimensional chain of blobs of size $D$, laterally confined within a disk of size $L$. Above the line, the chain of blobs becomes compact, and the bulge is, from the scaling perspective, better described by a an homogeneous polymer solution confined in a disk of size $L$ and thickness $D$. A third line III defined by $\overline{w \kappa}^{23 / 13}=\bar{\gamma}^{28 / 13}$ in the logarithmic representation, separates also tension 

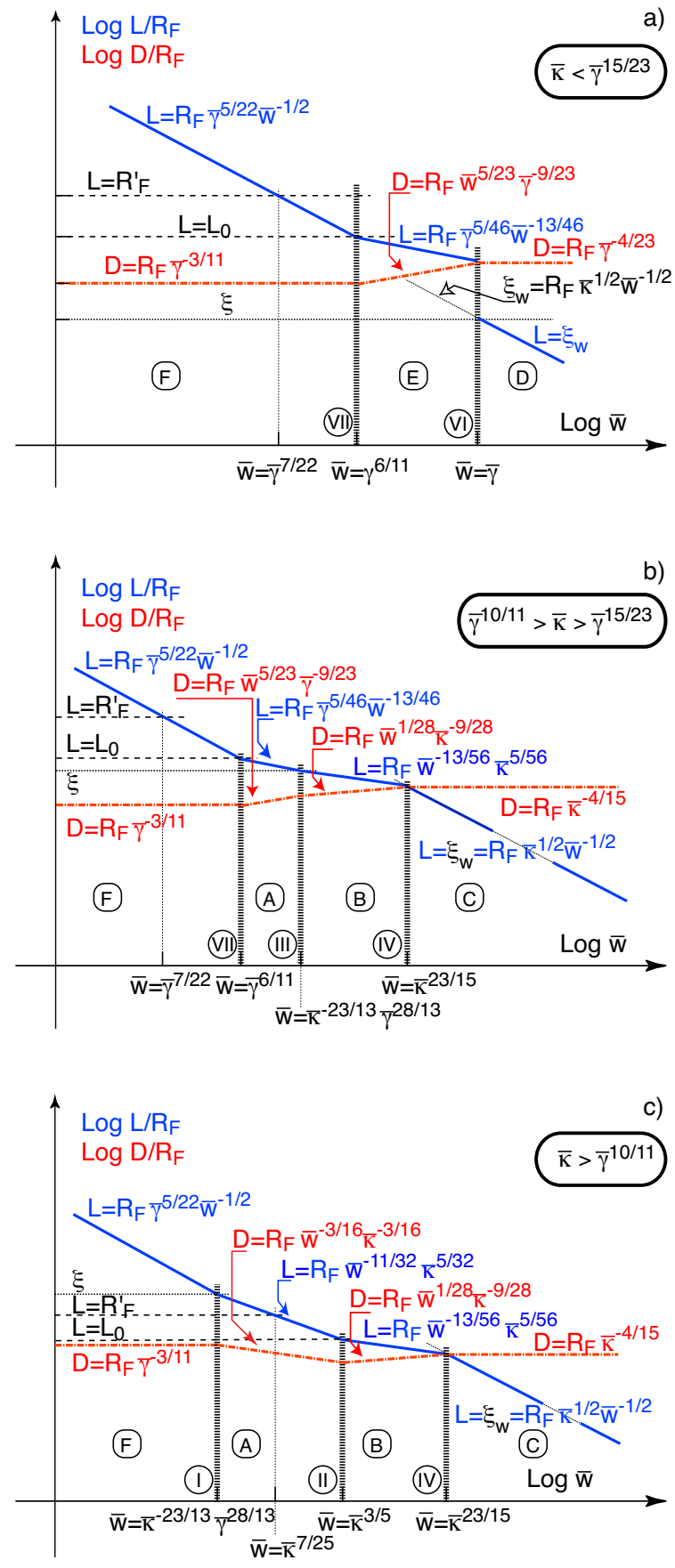

FIG. 7. (Color online) Variation of the bulge height $D$ and size $L$ as a function of the adhesion strength $\bar{w}$ in a logarithmic representation. a) Weak bending rigidities, $\bar{\kappa}<\bar{\gamma}^{15 / 23}$. b) Intermediate bending rigidities, $\bar{\gamma}^{10 / 11}>\bar{\kappa}>\bar{\gamma}^{15 / 23}$. c) Strong bending rigidities, $\bar{\kappa}>\bar{\gamma}^{10 / 11}$, see also Figure (3)

dominated and curvature dominated regions, on the line one has $L=\xi$. The fourth line IV defined by $\bar{w} \ll \bar{\kappa}^{23 / 15}$ separates shallow bulges $D \leq L$ and balloon bubbles $L \leq D$ in the domain above $\bar{\kappa}=\bar{\gamma}^{15 / 23}$ where curvature energy dominates. In the state diagram of Figure (6), line IV of slope $23 / 15$ where $L=D=\xi_{w}$, inter- sects the line $\ln \bar{w}=28 / 13 \ln \bar{\gamma}-23 / 13 \ln \bar{\kappa}$ at the point $(\ln \bar{\kappa}=15 / 23 \ln \bar{\gamma}, \ln \bar{w}=\ln \bar{\gamma})$. This is also the point of intersection of line VI, another important curve in the diagram, separating the balloon and the shallow bulge regimes in the domain where membrane tension dominates.

The swollen state diagram has thus six distinct regions, compare with the gaussian chain state diagram that displays four regions. For all the six regions, under the assumption that the membrane tension is large enough, $\bar{\gamma}>1$, the polymer chain is always confined, i.e. $D \leq R_{F}$. Region A corresponds thus to confined chains $\left(D \leq R_{F}\right)$ in shallow bulges $(D \leq L)$. Here, the thickness and patch size depend both on curvature elasticity and adhesion strength, but are independent of tension, $D \simeq R_{F}(\overline{\kappa w})^{-3 / 16}$ and $L \simeq R_{F} \bar{\kappa}^{5 / 32} \bar{w}^{-11 / 32}$. Region $\mathrm{B}$ hosts vertically and laterally confined chains $\left(D \leq R_{F}\right.$, $\left.L \leq L_{0}\right)$ in shallow bulges $(D \ll L)$. In this region, the equilibrium $D$ and $L$ values are independent of $\bar{\gamma}$, $L \simeq R_{F} \bar{w}^{-13 / 56} \bar{\kappa}^{5 / 56}$ and $D=R_{F} \bar{w}^{1 / 28} \bar{\kappa}^{-9 / 28}$. Above line VI, as for gaussian chain bulges, the neck size $L$ is fixed at $L=\xi_{w}$ and the bulge size at $D=R_{F} \bar{\kappa}^{-4 / 15}$. By crossing line $\mathrm{V}$ into region $\mathrm{D}$, the size of the balloon bulges becomes dependent of membrane tension, $D \simeq R_{F} \bar{\gamma}^{-4 / 23}$, but the size of the patch $L$ is still governed by the usual boundary condition balancing adhesion forces and curvature torque that defines the bulge neck size as $L=\xi_{w}$. This neck starts to open up at $\bar{w}=2 \bar{\gamma}$ close to line VI defined by $\bar{w}=\bar{\gamma}$ that one crosses into region $\mathrm{E}$, a shallow bulge region of laterally confined two dimensional chains, controlled by membrane tension, where $L \simeq R_{F} \bar{\gamma}^{-5 / 46} \bar{w}^{-13 / 46}$ and $D=R_{F} \bar{w}^{1 / 28} \bar{\kappa}^{-9 / 28}$. Below line VI the energy of lateral confinement of the chains is negligible and one recovers the shallow bulge height $D \simeq R_{F} \bar{\gamma}^{-3 / 11}$ and bulge size $L \simeq R_{F} \bar{\gamma}^{5 / 22} \bar{w}^{-1 / 2}$.

Also shown in Figure (6), the dashed lines indicate the crossover from laterally unconfined states of the chain below the lines to laterally confined chains. Note however that until one reaches lines II or VII, the energies of lateral confinement remain negligible. Note also that the minimum value $\bar{\gamma}$ that lines VII and VI can assume corresponds to $\bar{\gamma}=1$. For smaller values of $\bar{\gamma}$ the lines vanish from the diagram, with line I fixed for $\bar{\gamma}<1$ at the value $\ln \bar{w}=-\ln \bar{\kappa}$. The conclusions discussed above still hold, with the exception that in regions D, E and $\mathrm{F}$, that now merge into a single one, where the value of the height $\mathrm{D}$ is kept at the constant value $D \simeq R_{F}$ : the membrane tension in this case $\bar{\gamma}<1$ is not large enough to confine the polymer chain.

As for the gaussian chains we choose to display in Figure (7) the variation of the bulge height $D$ and size $L$ at constant elastic constant $\bar{\kappa}$ as a function of adhesion strength $\bar{w}$. Given the structure of the state diagram in Figure (6), three cases need to be considered. The first corresponds to weak elastic constants $\bar{\kappa}<\bar{\gamma}^{15 / 23}$, displayed in Figure (7a). The figure shows that the bulge height $D$ is first independent of the adhesion strength, then increases with adhesion reaching a second plateau. 
The patch size $L$ decreases continuously except at $\bar{w} \sim \bar{\gamma}$ where the so called kissing transition [29, 30] occurs. There, the size decreases from a finite value to $L \sim \xi_{w}$ within a narrow range $\bar{\gamma}<\bar{w}<2 \bar{\gamma}$. The second case corresponds to intermediate $\bar{\kappa}$ values $\bar{\gamma}^{10 / 11}>\bar{\kappa}>\bar{\gamma}^{15 / 23}$, displayed in Figure (7b), with similar variation trends for $D$ and $L$ as those of Figure (7a). For stronger elastic constants $\bar{\kappa}>\bar{\gamma}^{10 / 11}$, Figure (7c) shows that although $L$ decrease continuously as the adhesion strength increases, $D$ follows a non-monotonous variation in the regime of strong lateral confinement.

\section{Appendix B: Determination of the pressure profile}

We consider a polymer confined between two parallel planes, located respectively at $z=0$ and $z=D$, and attached near the lower plane at $(x=0, y=0, z \simeq b)$, with $b=2 \ell_{p}$ the statistical segment length (or Kuhn length), small compared with the separation $D$.

By considering an arbitrarily small deformation of the opposite surface $h(x, y)=D+\zeta(x, y)$, one can deduce the pressure field $p(x, y)$ by considering the isothermal work $\delta W$ associated to this deformation, and equal to the functional derivative of the free-energy $\mathcal{F}$ of the chain with respect to the distance $h(x, y)$ :

$\delta W=-\int \mathrm{d} x \mathrm{~d} y p(x, y) \zeta(x, y)=\int \mathrm{d} x \mathrm{~d} y \frac{\delta \mathcal{F}}{\delta h(x, y)} \zeta(x, y)$.

The calculation of the free energy $\mathcal{F}$ of a gaussian chain enclosed in a finite domain proceeds from solving the Edwards equation (or free diffusion) [18, 27],

$$
\begin{aligned}
& \frac{a b}{6}\left(\partial_{x}^{2}+\partial_{y}^{2}+\partial_{z}^{2}\right) \mathcal{G}(x, y, z ; N \mid 0,0, b ; 0)= \\
& \partial_{N} \mathcal{G}(x, y, z ; N \mid 0,0, b ; 0),
\end{aligned}
$$

while imposing absorbing boundary conditions $\mathcal{G}(x, y, z ; N \mid 0,0, b ; 0)=0$ on both surfaces:

$$
\begin{aligned}
& \forall(x, y, N) \\
& \mathcal{G}(x, y, 0 ; N \mid 0,0, b ; 0)=0 \text { and } \\
& \mathcal{G}(x, y, D+\zeta(x, y) ; N \mid 0,0, b ; 0)=0
\end{aligned}
$$

and with an initial condition localized on the grafting point at $N=0$ :

$$
\mathcal{G}(x, y, z ; 0 \mid 0,0, b ; 0)=\delta(x) \delta(y) \delta(z-b) .
$$

The diffusion coefficient of the Edwards equation is such that the gyration radius $R_{g}$ of a free chain of $N$ monomers equals precisely $N a b / 6$, with bond length $a$ and persistence $b$. Integration of $\mathcal{G}$ over the space variables $(x, y, z)$ gives the ratio between the number of configurations of a confined chain of length $N$ and the total number of configurations that the chain could adopt in the absence of any boundaries. Up to a constant independent of $\zeta(x, y), \mathcal{F}$ reads:

$$
\begin{gathered}
\mathcal{F}=-k_{B} T \ln \left[\int_{0}^{D+\zeta(x, y)} \mathrm{d} z \int \mathrm{d} x \mathrm{~d} y\right. \\
\mathcal{G}(x, y, z ; N \mid 0,0, b ; 0)] .
\end{gathered}
$$

Following ref [48, 49], we expand $\mathcal{G}$ in powers of $\zeta, \mathcal{G}=$ $\mathcal{G}_{0}+\mathcal{G}_{1} \ldots$, with $\mathcal{G}_{0} \sim \zeta^{0} \sim 1$ and $\mathcal{G}_{1} \sim \zeta^{1}$. $\mathcal{G}_{0}$ and $\mathcal{G}_{1}$ both obey the Edwards equation, with initial condition:

$$
\begin{aligned}
& \mathcal{G}_{0}(x, y, z ; 0 \mid 0,0, b ; 0)=\delta(x) \delta(y) \delta(z-b) \\
& \mathcal{G}_{1}(x, y, z ; 0 \mid 0,0, b ; 0)=0
\end{aligned}
$$

Inserting the expansion $\mathcal{G}=\mathcal{G}_{0}+\mathcal{G}_{1} \ldots$ at the vicinity of the upper boundary, one gets:

$$
\begin{gathered}
\mathcal{G}(x, y, D+\zeta(x, y) ; N \mid 0,0, b ; 0)= \\
\mathcal{G}(x, y, D ; N \mid 0,0, b ; 0)+ \\
\zeta(x, y) \partial_{z} \mathcal{G}(x, y, z=D ; N \mid 0,0, b ; 0)+\ldots=0
\end{gathered}
$$

leading to a set of boundary conditions:

$$
\begin{aligned}
& \mathcal{G}_{0}(x, y, 0 ; N \mid 0,0, b ; 0)=0 \\
& \mathcal{G}_{1}(x, y, 0 ; N \mid 0,0, b ; 0)=0 \\
& \mathcal{G}_{0}(x, y, D ; N \mid 0,0, b ; 0)=0 \\
& \mathcal{G}_{1}(x, y, D ; N \mid 0,0, b ; 0)= \\
& \quad-\zeta(x, y) \partial_{z} \mathcal{G}_{0}(x, y, z=D ; N \mid 0,0, b ; 0) .
\end{aligned}
$$

The leading term $\mathcal{G}_{0}$ corresponds to an ideal chain between two parallel plates. Separating variables, $\mathcal{G}_{0}$ reads:

$$
\begin{aligned}
\mathcal{G}_{0}(x, y, z ; n \mid & \left.x^{\prime}, y^{\prime}, z^{\prime} ; n^{\prime}\right)= \\
& \mathcal{G}^{(x)}\left(x-x^{\prime} ; n-n^{\prime}\right) \times \\
& \mathcal{G}^{(y)}\left(y-y^{\prime} ; n-n^{\prime}\right) \times \\
& \mathcal{G}^{(z)}\left(z, z^{\prime} ; n-n^{\prime}\right), \\
\mathcal{G}^{(x)}\left(x-x^{\prime} ; n\right)= & \sqrt{\frac{3}{2 \pi a b n} \times} \\
& \exp \left(-\frac{3\left(x-x^{\prime}\right)^{2}}{2 n a b}\right) ; \\
\mathcal{G}^{(y)}\left(y-y^{\prime} ; n\right)= & \sqrt{\frac{3}{2 \pi a b n} \times} \times \\
& \exp \left(-\frac{3\left(y-y^{\prime}\right)^{2}}{2 n a b}\right) ; \\
\mathcal{G}^{(z)}\left(z, z^{\prime} ; n\right)= & \frac{2}{D} \sum_{j=1}^{\infty} \sin \left(\frac{j \pi z}{D}\right) \times \\
& \sin \left(\frac{j \pi z^{\prime}}{D}\right) \times \\
& \exp \left(-\frac{j^{2} \pi^{2} a b}{6 D^{2}} n\right) .
\end{aligned}
$$


The perturbative term $\mathcal{G}_{1}$ originates entirely from the contribution of the boundaries, where $\mathcal{G}_{1}$ is explicitly known (Dirichlet boundary condition). $\mathcal{G}_{1}$ is:

$$
\begin{aligned}
& \mathcal{G}_{1}(x, y, z ; N \mid 0,0, b ; 0)= \\
& -\frac{a b}{6} \int \mathrm{d} x^{\prime} \mathrm{d} y^{\prime} \int_{0}^{N} \mathrm{~d} n \\
& \quad \partial_{z^{\prime}} \mathcal{G}_{0}\left(x, y, z ; N-n \mid x^{\prime}, y^{\prime}, z^{\prime}=D ; 0\right) \times \\
& \mathcal{G}_{1}\left(x^{\prime}, y^{\prime}, D ; n \mid 0,0, b ; 0\right)= \\
& \frac{a b}{6} \int \mathrm{d} x^{\prime} \mathrm{d} y^{\prime} \int_{0}^{N} \mathrm{~d} n \\
& \quad \partial_{z^{\prime}} \mathcal{G}_{0}\left(x, y, z ; N-n \mid x^{\prime}, y^{\prime}, D ; 0\right) \times \\
& \zeta\left(y^{\prime}, z^{\prime}\right) \partial_{z^{\prime}} \mathcal{G}_{0}\left(x^{\prime}, y^{\prime}, z^{\prime}=D ; n \mid 0,0, b ; 0\right)
\end{aligned}
$$

The above expression shows that the determination of the pressure profile is subordinated to the entire knowledge of the solution $\mathcal{G}_{0}$ of the unperturbed case. At the order $\zeta$, the free energy is:

$$
\begin{aligned}
\mathcal{F}= & -k_{B} T \ln \left\{\int \mathrm{d} x \mathrm{~d} y \int_{0}^{D} \mathrm{~d} z \mathcal{G}_{0}(x, y, z ; N \mid 0,0, b ; 0)+\right. \\
& \left.\mathcal{G}_{1}(x, y, z ; N \mid 0,0, b ; 0)\right\} \\
\simeq & -k_{B} T \ln \left\{\int \mathrm{d} x \mathrm{~d} y \int_{0}^{D} \mathrm{~d} z \mathcal{G}_{0}(x, y, z ; N \mid 0,0, b ; 0)\right\} \\
& -k_{B} T \frac{\int \mathrm{d} x \mathrm{~d} y \int_{0}^{D} \mathrm{~d} z \mathcal{G}_{1}(x, y, z ; N \mid 0,0, b ; 0)}{\int \mathrm{d} x \mathrm{~d} y \int_{0}^{D} \mathrm{~d} z \mathcal{G}_{0}(x, y, z ; N \mid 0,0, b ; 0)}
\end{aligned}
$$

The first term can be easily calculated and leads to the expected scaling result $\mathcal{F} \sim k_{B} T R_{g}^{2} / D^{2}$ for an ideal chain confined in the $z$-direction. We deduce the pressure field $p(x, y)$ from the second term, identifying it with eq. (B1), to get:

$$
\begin{aligned}
p\left(x^{\prime}, y^{\prime}\right)= & k_{B} T \frac{a b}{6}\left\{\int_{0}^{N} \mathrm{~d} n \iiint \mathrm{d} x \mathrm{~d} y \mathrm{~d} z\right. \\
& {\left[\partial_{z^{\prime}} \mathcal{G}_{0}\left(x, y, z ; N \mid x^{\prime}, y^{\prime}, z^{\prime}=D ; N-n\right)\right.} \\
& \left.\left.\partial_{z^{\prime}} \mathcal{G}_{0}\left(x^{\prime}, y^{\prime}, z^{\prime}=D ; n \mid 0,0, b ; 0\right)\right]\right\} \\
& \times\left[\iiint \mathrm{d} x \mathrm{~d} y \mathrm{~d} z \mathcal{G}_{0}(x, y, z ; N \mid 0,0, b ; 0)\right]^{-1}
\end{aligned}
$$

Eq. (B17) has a simple interpretation, illustrated on Fig. 8. The pressure at an arbitrary point M' of the confining domain is given by the sum over all the paths between the origin $\mathrm{M}_{0}$ of the chain and $\mathrm{M}$ ', and leaving M' for the endpoint $M$ of the chain. This sum of paths is weighted by a product of gradients of the Green function associated to the domain, and normalized by a sum over all paths between the origin $\mathrm{M}_{0}$ and $\mathrm{M}$.

We now proceed with the explicit expressions provided in eq. (B9). It is customary to retain only the first term

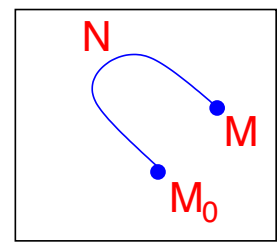

Normalization

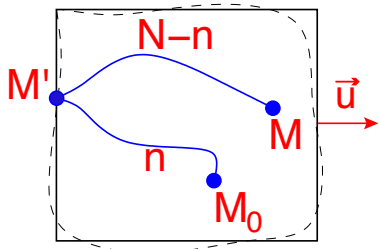

Pressure at $\mathrm{M}^{\prime}$
FIG. 8. Schematic representation of the sum of paths leading the inhomogeneous pressure field.

$j=1$ of the Fourier sum in $G^{(z)}\left(z, z^{\prime} ; N\right)$, whenever the ratio $D / R_{g}$ is much smaller than 1 , meaning that the chain is strongly confined (lowest level, or ground state dominance approximation). With shallow bulges in mind, we make this approximation. The integration over $x$ and $y$ of the normalized gaussian integrals is trivial and we obtain, noting $r^{\prime 2}=x^{\prime 2}+y^{\prime 2}$ :

$$
\begin{gathered}
\iiint \mathrm{d} x \mathrm{~d} y \mathrm{~d} z \mathcal{G}_{0}(x, y, z ; N \mid 0,0, b ; 0)= \\
\frac{4}{\pi} \sin \left(\frac{\pi b}{D}\right) \exp \left(-\frac{\pi^{2} R_{g}^{2}}{D^{2}}\right) ; \\
\iiint \mathrm{d} x \mathrm{~d} y \mathrm{~d} z \mathcal{G}_{1}(x, y, z ; N \mid 0,0, b ; 0)= \\
\frac{12}{a b D^{3}} \sin \left(\frac{\pi b}{N}\right) \exp \left(-\frac{\pi^{2} R_{g}^{2}}{D^{2}}\right) \times \\
\int_{0}^{N} \frac{\mathrm{d} n}{n} \exp \left(-\frac{3 r^{\prime 2}}{2 n a b}\right) .
\end{gathered}
$$

The integral over $n$ reduces to a standard expression:

$$
\int_{r^{\prime 2} /\left(4 R_{g}^{2}\right)}^{\infty} \mathrm{d} y \frac{e^{-y}}{y}=\mathrm{E}_{1}\left(\frac{r^{\prime 2}}{4 R_{g}^{2}}\right)=\Gamma\left(0, \frac{r^{\prime 2}}{4 R_{g}^{2}}\right),
$$

where $\mathrm{E}_{1}(x)$ and $\Gamma(0, x)$ designates respectively the integral exponential and the incomplete Gamma function [50].

Altogether, the final expression for the pressure field associated with a bulge of height $D$ is:

$$
p(x, y)=\frac{\pi k_{B} T}{2 D^{3}} \Gamma\left(0, \frac{r^{2}}{4 R_{g}^{2}}\right)
$$

At the vertical of the grafting point, the pressure diverges logarithmically with $r^{\prime}$. This singularity, which can be easily integrated over, and has only mild consequences for the determination of the bulge profile. We can alternatively cut-off the singularity at $\Gamma(0, b)$, as the ideal chain model breaks down on length scales smaller than $b$. This regularization does not lead to any observable numerical difference. In the opposite limit, the pressure decays rapidly with $r^{\prime}$, to become negligible on distances larger than the gyration radius. Provided the height $h(r)$ remains close to $D$ on such distances, we expect the parallel plane approximation to be quantitatively satisfactory. 


\section{Appendix C: Determination of the membrane mechanical response}

A rotationally invariant profile is described by a height function $h(r)$. In the Monge approximation, gradients of $h(r)$ are weak and the mechanical equilibrium requires

$$
\kappa \Delta \Delta h-\gamma \Delta h=p(r)
$$

with boundary conditions at $r=L$. This linear equation can be solved by means of a Green function $G_{\gamma, \kappa, L}\left(r, r^{\prime}\right)$ such that

$$
\left(\kappa \Delta_{r} \Delta_{r}-\gamma \Delta_{r}\right) G_{\gamma, \kappa, L}\left(r, r^{\prime}\right)=\frac{\delta\left(r-r^{\prime}\right)}{r} .
$$

The resulting Green function $G_{\gamma, \kappa, L}$ makes it possible to express the profile $h(r)$ as a convolution of the pressure field

$$
h(r)=\int_{0}^{L} \mathrm{~d} r^{\prime} r^{\prime} G_{\gamma, \kappa, L}\left(r, r^{\prime}\right) p\left(r^{\prime}\right)
$$

but it also gives convenient expressions for its derivatives

$$
\begin{aligned}
h^{\prime}(r) & =\int_{0}^{L} \mathrm{~d} r^{\prime} r^{\prime} \partial_{r} G_{\gamma, \kappa, L}\left(r, r^{\prime}\right) p\left(r^{\prime}\right) \\
\Delta h(r) & =\int_{0}^{L} \mathrm{~d} r^{\prime} r^{\prime} \Delta_{r} G_{\gamma, \kappa, L}\left(r, r^{\prime}\right) p\left(r^{\prime}\right)
\end{aligned}
$$

found in the expressions defining the free-energy.

The functions $G_{\gamma, \kappa, L}\left(r, r^{\prime}\right)$ describe the elastic response of a circular patch of membrane to a uniform force exerted at a distance $r^{\prime}$ from its center. The membrane mechanical response results from both membrane properties and from the value of the membrane-surface adhesion parameter. The membrane mechanical response is thus described by the energy scale $T$, a characteristic length $L$ and two internal, dimensionless parameters $\bar{\kappa}=\kappa / T$ and $\nu=\sqrt{\gamma L^{2} / \kappa}=L / \xi$. The adhesion properties of the surface-membrane interaction manifests themselves only through $L$. The scaling function fulfills the following requirements:

$$
\kappa \Delta \Delta G_{\gamma, \kappa, L}\left(r, r^{\prime}\right)-\gamma \Delta G_{\gamma, \kappa, L}\left(r, r^{\prime}\right)=\frac{\delta\left(r-r^{\prime}\right)}{r} ;
$$

with two boundary conditions at $r=0$ :

$$
\begin{aligned}
\lim _{r \rightarrow 0} \frac{\partial G_{\gamma, \kappa, L}\left(r, r^{\prime}\right)}{\partial r} & =0 ; \\
\lim _{r \rightarrow 0} \frac{\partial^{3} G_{\gamma, \kappa, L}\left(r, r^{\prime}\right)}{\partial r^{3}} & =0,
\end{aligned}
$$

and two boundary conditions at $r=L$ :

$$
\begin{aligned}
G_{\gamma, \kappa, L}\left(L, r^{\prime}\right) & =0 \\
\left.\frac{\partial G_{\gamma, \kappa, L}\left(r, r^{\prime}\right)}{\partial r}\right|_{r=L} & =0 .
\end{aligned}
$$

Two particular cases of interest are the opposite situations of vanishing bending modulus $\kappa=0$ and surface tension $\gamma=0$. If $\kappa=0$, we have:

$$
\begin{aligned}
& G_{\gamma, 0, L}\left(r, r^{\prime}\right)=\gamma^{-1} \ln \left(\frac{L}{r^{\prime}}\right), \text { if } r<r^{\prime} \\
& G_{\gamma, 0, L}\left(r, r^{\prime}\right)=\gamma^{-1} \ln \left(\frac{L}{r}\right), \text { if } r>r^{\prime}
\end{aligned}
$$

we observe that the boundary conditions are less demanding as the second derivative at $r=L$ does not need to be continuous.

$$
\begin{aligned}
& \text { If } \gamma=0, G \text { becomes } \\
& \qquad \begin{aligned}
G_{0, \kappa, L}\left(r, r^{\prime}\right)= & \frac{L^{2}}{8}\left(\left(1+\frac{r^{2}}{L^{2}}\right)\left(1-\frac{r^{\prime 2}}{L^{2}}\right)+\right. \\
& \left.\left(\frac{r^{2}}{L^{2}}+\frac{r^{\prime 2}}{L^{2}}\right) \ln \left(\frac{r^{\prime 2}}{L^{2}}\right)\right) \text { if } r<r^{\prime} \\
G_{0, \kappa, L}\left(r, r^{\prime}\right)= & \frac{L^{2}}{8}\left(\left(1-\frac{r^{2}}{L^{2}}\right)\left(1+\frac{r^{\prime 2}}{L^{2}}\right)+\right. \\
& \left.\left(\frac{r^{2}}{L^{2}}+\frac{r^{\prime 2}}{L^{2}}\right) \ln \left(\frac{r^{2}}{L^{2}}\right)\right) \text { if } r>r^{\prime}
\end{aligned}
\end{aligned}
$$

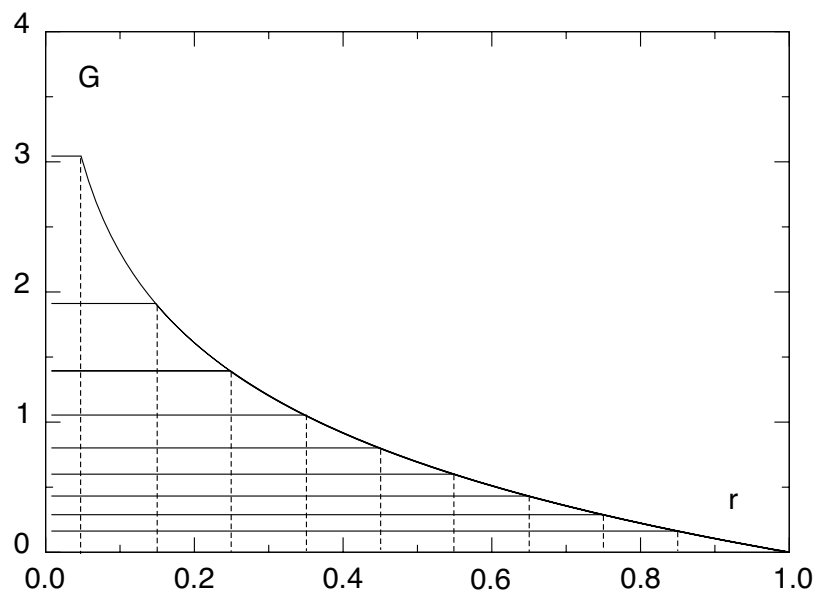

FIG. 9. Green function $G_{\gamma=1, \kappa=0, L=1}\left(r, r^{\prime}\right)$ as a function of $r$, for values of $r^{\prime}$ equal to $0.05,0.15,0.25,0.35,0.45,0.55,0.65$, 0.75 and 0.85 .

Figure (9) shows a set of $G_{\gamma, 0, L}\left(r, r^{\prime}\right)$ for evenly spaced values of $r^{\prime}, L=1, \kappa=0$ and $\gamma=1$. The profiles are flat for $r<r^{\prime}$ and logarithmic for $r>r^{\prime}$. As expected, the membrane profile can stand a non vanishing slope near the $\operatorname{rim} r=L$ of the bubble, in a situation closely related to the well known wetting profiles. Figure (10) shows a set of $G_{0, \kappa, L}\left(r, r^{\prime}\right)$ for evenly spaced values of $r^{\prime}, L=1$, $\gamma=0$ and $\kappa=1$.

Whenever curvature is present the second derivative (or Laplacian) of $G$ with respect to $r$ must exist. This 


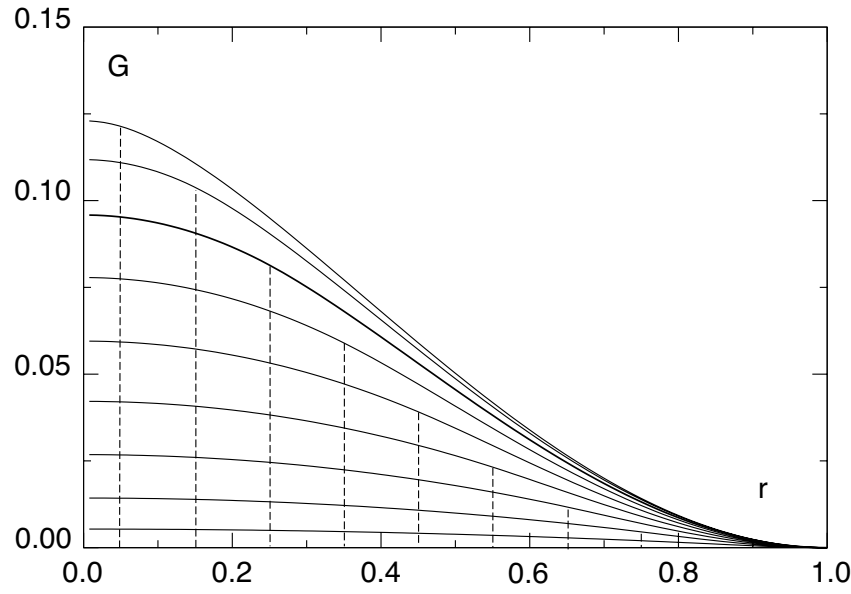

FIG. 10. Green function $G_{\gamma=0, \kappa=1, L=1}\left(r, r^{\prime}\right)$ as a function of $r$, for values of $r^{\prime}$ equal to $0.05,0.15,0.25,0.35,0.45,0.55,0.65$, 0.75 and 0.85 .

implies that both $G\left(r, r^{\prime}\right)$ and $\partial_{r} G\left(r, r^{\prime}\right)$ vanish at $r=L$. Introducing the characteristic length scale $\xi=\sqrt{\kappa / \gamma}$, and dimensionless space variables $x=r / \xi$ and $x^{\prime}=r^{\prime} / \xi$, the Green equation becomes

$$
\left(\Delta_{x}^{2}-\Delta_{x}\right) G_{\gamma, \kappa, L}\left(x \xi, x^{\prime} \xi\right)=\frac{1}{\gamma} \frac{\delta\left(x-x^{\prime}\right)}{x},
$$

This suggest the following scaling expression for $G_{\gamma, \kappa, L}\left(r, r^{\prime}\right)$ :

$$
G_{\gamma, \kappa, L}\left(r, r^{\prime}\right)=\frac{1}{\gamma} \bar{G}_{L / \xi}\left(\frac{r}{\bar{\xi}}, \frac{r^{\prime}}{\xi}\right)=\frac{L^{2}}{\nu^{2} T \bar{\kappa}} \bar{G}_{\nu}\left(\frac{r \nu}{L}, \frac{r^{\prime} \nu}{L}\right)
$$

with $\nu=L / \xi=\sqrt{\gamma L^{2} / \kappa}$,

$$
\Delta_{x}\left(\Delta_{x}-1\right) G_{\nu=L / \xi}\left(x, x^{\prime}\right)=\frac{\delta\left(x-x^{\prime}\right)}{x},
$$

defining a family of $\nu$-dependent Green functions. The factorized form of the differential operator gives a hint for finding the four linearly independent solutions of the fourth order linear differential equation; the two first one are the constant and $\ln (x)$, the two others are the modified Bessel functions $\mathrm{K}_{0}(x)$ and $\mathrm{I}_{0}(x)$. $\bar{G}\left(x, x^{\prime}\right)=$ $\alpha\left(x^{\prime}\right)+\beta\left(x^{\prime}\right) \ln (x)+\gamma\left(x^{\prime}\right) \mathrm{K}_{0}(x)+\delta\left(x^{\prime}\right) \mathrm{I}_{0}(x)$ if $x<x^{\prime}$ and $\bar{G}\left(x, x^{\prime}\right)=\alpha^{\prime}\left(x^{\prime}\right)+\beta^{\prime}\left(x^{\prime}\right) \ln (x)+\gamma^{\prime}\left(x^{\prime}\right) \mathrm{K}_{0}(x)+\delta^{\prime}\left(x^{\prime}\right) \mathrm{I}_{0}(x)$ if $x>x^{\prime}$.

The boundary $x=L$ provides two conditions $\bar{G}_{\nu}\left(\nu, x^{\prime}\right)=0$ and $\left.\partial_{x} \bar{G}_{\nu}\left(x, x^{\prime}\right)\right|_{x=\nu}=0$, from where the $\nu$ dependence explicitly originates. The boundary $x=0$ is more subtle as 0 is a singular point of the differential equation. It is not possible to enforce independently $\bar{G}_{\nu}\left(0, x^{\prime}\right)$ and $\partial_{x} \bar{G}_{\nu}\left(0, x^{\prime}\right)$. However, because $\bar{G}_{\nu}$ is the radial part of a rotationally invariant solution, we must have $\partial_{x} \bar{G}_{\nu}\left(x, x^{\prime}\right)=\left.\partial_{x x x}^{3} \bar{G}_{\nu}\left(x, x^{\prime}\right)\right|_{x=0}=0$, giving another two conditions.

The continuity relation at $x=x^{\prime}$ implies $\bar{G}_{\nu}\left(x, x^{\prime}\right)$, $\partial_{x} \bar{G}_{\nu}\left(x, x^{\prime}\right), \partial_{x x}^{2} \bar{G}_{\nu}\left(x, x^{\prime}\right)$ continuous at $x=x^{\prime}$ and $\lim _{x \rightarrow\left(x^{\prime}+\right)} \partial_{x x x}^{3} \bar{G}_{\nu}\left(x, x^{\prime}\right)-\lim _{x \rightarrow\left(x^{\prime}-\right)} \partial_{x x x}^{3} \bar{G}_{\nu}\left(x, x^{\prime}\right)=$ $1 / x^{\prime}$. Altogether, these eight conditions suffice for determining unambiguously $\bar{G}_{\nu}$. The result is:

$$
\begin{aligned}
\bar{G}_{\nu}\left(x, x^{\prime}\right)= & \ln \left[\frac{\nu}{x^{\prime}}\right]+\frac{\mathrm{I}_{0}\left(x^{\prime}\right)-\mathrm{I}_{0}(\nu)}{\nu \mathrm{I}_{1}(\nu)}+ \\
& \frac{\mathrm{I}_{0}(x)}{\mathrm{I}_{1}(\nu)}\left[\frac{1}{\nu}-\left(\mathrm{I}_{1}(\nu) \mathrm{K}_{0}\left(x^{\prime}\right)+\right.\right. \\
& \left.\left.\mathrm{K}_{1}(\nu) \mathrm{I}_{0}\left(x^{\prime}\right)\right)\right] \text { if } x<x^{\prime} ; \\
\bar{G}_{\nu}\left(x, x^{\prime}\right)= & \ln \left[\frac{\nu}{x}\right]+\frac{\mathrm{I}_{0}(x)-\mathrm{I}_{0}(\nu)}{\nu \mathrm{I}_{1}(\nu)}+ \\
& \frac{\mathrm{I}_{0}\left(x^{\prime}\right)}{\mathrm{I}_{1}(\nu)}\left[\frac{1}{\nu}-\left(\mathrm{I}_{1}(\nu) \mathrm{K}_{0}(x)+\right.\right. \\
& \left.\left.\mathrm{K}_{1}(\nu) \mathrm{I}_{0}(x)\right)\right] \text { if } x>x^{\prime} ;
\end{aligned}
$$

We observe that $\bar{G}_{\nu}\left(x, x^{\prime}\right)=\bar{G}_{\nu}\left(x^{\prime}, x\right)$. In checking that this Green function fulfills all the desired properties, it is useful to remember that $\mathrm{I}_{0}^{\prime}(x)=\mathrm{I}_{1}(x)$, $\mathrm{K}_{0}^{\prime}(x)=-\mathrm{K}_{1}(x)$, that $\mathrm{I}_{0}$ and $\mathrm{K}_{0}$ solve the modified Bessel equation $x^{2} f^{\prime \prime}(x)+x f^{\prime}(x)-x^{2} f(x)=0$ and obey the Wronskian relation $\mathrm{I}_{0}(x) \mathrm{K}_{1}(x)+\mathrm{I}_{1}(x) \mathrm{K}_{0}(x)=1 / x$.

When both curvature and tension are present, the curves depend continuously on $\nu$ and interpolate smoothly between the two aforementioned cases $\nu=0$ and $\nu=\infty$ respectively. For instance Figure (11) presents the case $\nu=1$.

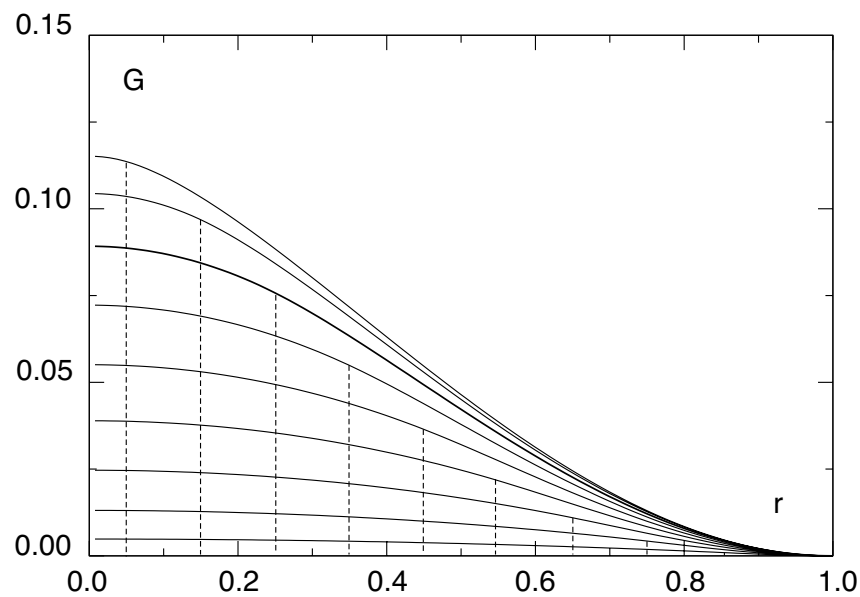

FIG. 11. Green function $G_{\gamma=1, \kappa=1, L=1}\left(r, r^{\prime}\right)$ as a function of $r$, for values of $r^{\prime}$ equal to $0.05,0.15,0.25,0.35,0.45,0.55,0.65$, 0.75 and 0.85 . 
Appendix D: Profile with fixed patch radius $L$ and comparison with scaling results

\section{Profiles with fixed patch radius}

In our presentation of the mechanical response of the membrane, we introduced the ratio $\nu=L / \xi$, related to the competition between curvature and tension. Because in our numerical determination of the profile, $L$ plays a prominent role at the expense of the surface energy $w$, we use $L$ as our main length scale instead of $R_{g}$. As discussed, in the first section, the precise shape of the bubble actually depends on three dimensionless parameters. Two of them were already introduced in the course of defining the Green functions: $\nu$ and $\bar{\kappa}$. The third one is provided by the ratio $\mu=R_{g} / L$. The free-energy then reads:

$$
\begin{aligned}
\mathcal{F}= & \pi \bar{\kappa} T \int_{0}^{1} y \mathrm{~d} y\left[\nu^{2}(\nabla \bar{H}(y))^{2}+\left(\Delta_{y} \bar{H}(y)\right)^{2}\right] \\
& +\mathcal{F}_{\text {chain }}+\frac{\bar{\kappa} T}{2}\left[\Delta_{y} \bar{H}(1)\right]^{2} \\
\mathcal{F}_{\text {chain }}= & -2 \pi \int_{0}^{1} y \mathrm{~d} y \frac{\pi T}{2 \bar{H}(0)^{3}} \Gamma\left(0, \frac{y^{2}}{4 \mu^{2}}\right)
\end{aligned}
$$

and the profile $\bar{H}_{\nu, \mu, \bar{\kappa}}(x)$ resulting from the minimization is related to the true profile $h_{\gamma, \kappa, L, R_{g}}(r)$ by

$$
h_{\gamma, \kappa, L, R_{g}}(r)=L \bar{H}_{\nu, \mu, \bar{\kappa}}\left(\frac{r}{L}\right)
$$

The height $D$ of the bubble, the adhesion parameter $w$ and the pressure field $p(r ; D)$ all follow:

$$
\begin{aligned}
D & =L \bar{H}_{\nu, \mu, \bar{\kappa}}(0) \\
w & =\frac{\bar{\kappa} T}{2 L^{2}}\left[\left.\Delta_{x} \bar{H}_{\mu, \nu, \bar{\kappa}}(y)\right|_{y=1}\right]^{2} \\
p(r ; D) & =\frac{\pi T}{2 L^{3} \bar{H}_{\mu, \nu, \bar{\kappa}}(0)^{3}} \Gamma\left(0, \frac{r^{2}}{4 L^{2} \mu^{2}}\right)
\end{aligned}
$$

\section{Comparison with scaling results}

We compare the output of our self-consistent calculations with the scaling predictions. In the curvature dominated regime, one has:

$$
\begin{aligned}
\bar{H}(0) & \simeq \mu^{1 / 2} \bar{\kappa}^{-1 / 4}=D / L ; \\
\mathcal{F} & \simeq T \bar{\kappa}^{1 / 2} \mu ; \\
\bar{w} & \simeq \mu^{3} \kappa^{1 / 2} .
\end{aligned}
$$

In the tension dominated regime, the scaling behaviors are

$$
\begin{aligned}
\bar{H}(0) & \simeq \mu \bar{\gamma}^{-1 / 4} ; \\
\mathcal{F} & \simeq T \bar{\kappa}^{1 / 2} \mu \nu=T \bar{\gamma}^{1 / 2} ; \\
\bar{w} & \simeq \mu^{2} \bar{\gamma}^{1 / 2} .
\end{aligned}
$$

A test of these scaling relations is shown in Figure (12) and (13).

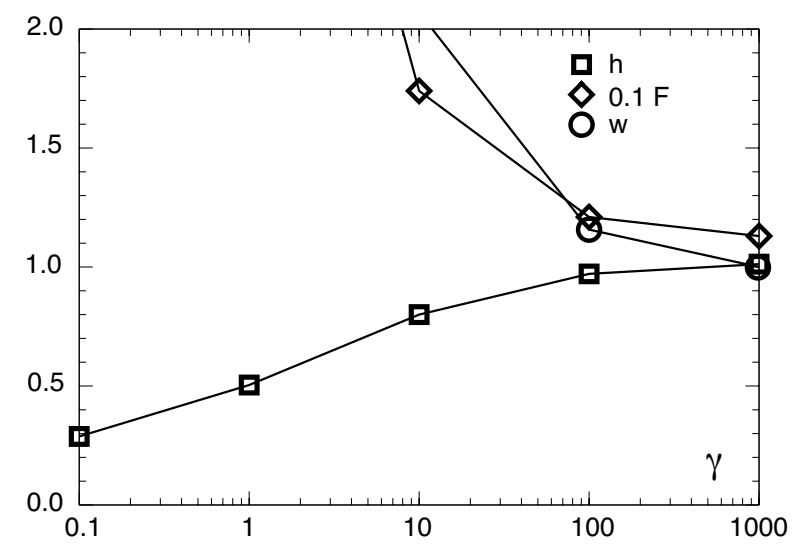

FIG. 12. Test of the scaling relations at large $\bar{\gamma}$. The ratios $\bar{H}(0) \mu^{-1} \bar{\gamma}^{1 / 4}$ (labeled "h"), $0.1 \mathcal{F} T^{-1} \bar{\gamma}^{-1 / 2}$ (labeled " $0.1 \mathrm{~F}$ ") and $\bar{w} \mu^{-2} \bar{\gamma}^{-1 / 2}$ (labeled "w") must tend towards a constant at large $\bar{\gamma}$, which happens for $\bar{\gamma} \geq 100$.

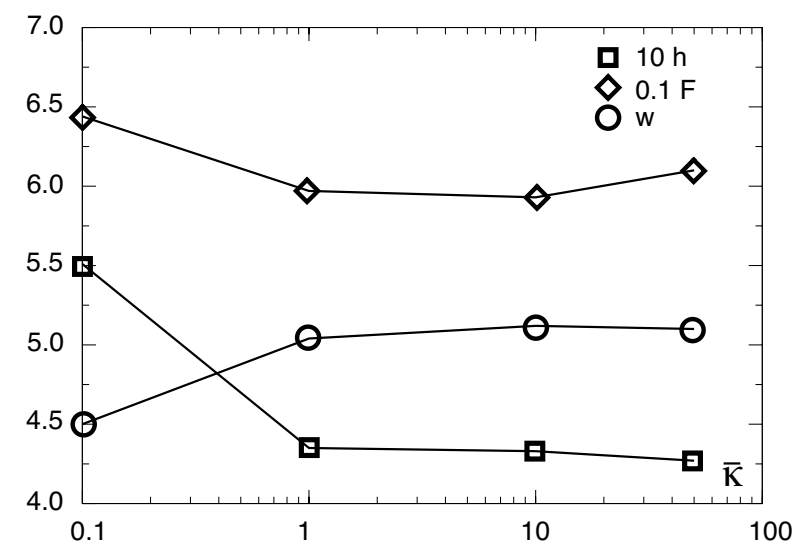

FIG. 13. Test of the scaling relations at large $\bar{\kappa}$. The ratios $10 \bar{H}(0) \mu^{-1 / 2} \bar{\kappa}^{1 / 4}$ (labeled "10h"), $0.1 \mathcal{F} T^{-1} \mu^{-1} \bar{\kappa}^{-1 / 2}$ (labeled " $0.1 \mathrm{~F}$ ") and $\bar{w} \mu^{-3} \bar{\kappa}^{-1 / 2}$ (labeled "w") should go to a constant value, as seen for $\bar{\kappa}$ comprised between 1 and 50 . 
[1] B. Alberts, A. Jonhson, J. Lewis, M. Raff, K. Roberts, and P. Walter, Molecular Biology of the Cell (Garland Science, Taylor and Francis, 2008).

[2] V. Frette, I. Tsafrir, M. Guedeau-Boudeville, L. Jullien, D. Kandel, and J. Stavans, Physical Review Letters 83, 2465 (1999).

[3] M. Cates, Nature 351, 102 (1991).

[4] H. Ringsdorf, J. Venzmer, and F. Winnik, Angewandte Chemie-International Edition In English 30, 315 (1991).

[5] R. Joannic, L. Auvray, and D. Lasic, Physical Review Letters 78, 3402 (1997).

[6] G. Bouglet and C. Ligoure, European Physical Journal B 9, 137 (1999).

[7] B. Yang, J. Lal, P. Richetti, C. M. Marques, W. Russel, and R. Prud'homme, Langmuir 17, 5834 (2001).

[8] Y. Yang, R. Prudhomme, K. McGrath, P. Richetti, and C. M. Marques, Physical Review Letters 80, 2729 (1998).

[9] H. Warriner, S. Idziak, N. Slack, P. Davidson, and C. Safinya, Science 271, 969 (1996).

[10] J. Vandepas, T. Olsthoorn, F. Schepers, C. Devries, and C. Buytenhek, Colloids and Surfaces A-Physicochemical and Engineering Aspects 85, 221 (1994).

[11] D. D. Lasic and Y. Barenholz, Handbook of nonmedical applications of liposomes (CRC Press, Boca Raton, 1996).

[12] D. D. Lasic and D. Papahadjopoulos, Medical applications of liposomes (Elsevier, Amsterdam, 1998).

[13] G. Gregoriadis, Targeting of drugs 6: strategies for stealth therapeutic systems, vol. v. 300 (Plenum Press, New York, 1998).

[14] H. Lodish, A. Berk, S. L. Zipursky, P. Matsudaira, D. Baltimore, and J. Darnell, Molecular Biology of the Cell (Feeman, 2000), 4th ed.

[15] K. Sengupta and L. Limozin, Phys. Rev. Lett. 104, 088101 (2010).

[16] W. Helfrich, Z. Naturforsch. C 28, 693 (1973).

[17] S. Safran, Statistical Thermodynamics of Surfaces, Interfaces and Membranes (Addison-Wesley, Reading, MA, 1994).

[18] P. G. de Gennes, Scalig concepts in polymer physics (Cornell University Press, 1979).

[19] T. Bickel and C. M. Marques, Journal of Nanosciences and Nanotechnology 6, 2386 (2006).

[20] M.-L. Hisette, P. Haddad, T. Gisler, C. M. Marques, and A. P. Schröder, Soft Matter 4, 828 (2008).

[21] A. Schröder, private communication.

[22] G. Subramanian, D. R. M. Williams, and P. A. Pincus, EPL (Europhysics Letters) 29, 285 (1995).

[23] G. Subramanian, D. Williams, and P. Pincus, Macromolecules 29, 4045 (1996).

[24] P. S. Swain and D. Andelman, Langmuir 15, 8902 (1999).

[25] An accurate treatment of thermal membrane undulations shows the need of distinguishing between a microscopic and an effective macroscopic surface tension parameter [47]. In our framework, the membrane is a purely mechanical object with a well-defined surface tension $\gamma$, that we associate to the macroscopic $\tau$ of ref. [47].

[26] E. F. Casassa and Y. Tagami, Macromolecules 2, 14 (1969).

[27] M. Doi and S. Edwards, The Theory of Polymer Dynamics (Oxford University Press, 1986).

[28] L. D. Landau, E. M. Lifshits, A. M. Kosevich, and L. P. Pitaevskiı, Theory of elasticity, vol. v. 7 of Course of theoretical physics (Pergamon Press, Oxford, 1986), 3rd ed.

[29] X. Michalet, F. Julicher, B. Fourcade, U. Seifert, and D. Bensimon, Recherche 25, 1012 (1994).

[30] B. Fourcade, L. Miao, M. Rao, M. Wortis, and R. Zia, Physical Review E 49, 5276 (1994).

[31] D. Schaefer, J. Joanny, and P. Pincus, Macromolecules 13, 1280 (1980).

[32] H. Nakanishi, Journal de Physique 48, p979 (1987).

[33] F. Brochard-Wyart, T. Tanaka, N. Borghi, and P.-G. de Gennes, P. G. Gennes, Langmuir 21, 4144 (2005).

[34] H. Isambert, Physical Review Letters 80, 3404 (1998).

[35] M.-C. Kim and D. Williams, Macromolecules 43, 3090 (2010).

[36] Udo Seifert, Karin Berndl and Reinhard Lipowsky, Phys.Rev.A 44, 1182 (1991).

[37] C. Tordeux, J.-B. Fournierand P. Galatola, Phys. Rev. E 65, $041912(2002)$

[38] D. Norouzi, M. M. Müller, and M. Deserno, Phys. Rev. E 74, 061914 (2006).

[39] J. N. Israelachvili, Intermolecular and Surface Forces (Elsevier, 1992).

[40] R. Capovilla and J. Guven, Phys. Rev. E 66, 041604 (2002).

[41] J.-B. Fournier, Soft Matter 3, 883 (2007).

[42] See EPAPS Document No. [number will be inserted by publisher] for [give brief description of material].

[43] S. B. Smith, L. Finzi, and C. Bustamante, Science 258, 1122 (1992).

[44] J. F. Marko and E. D. Siggia, Macromolecules 28, 8759 (1995).

[45] P. S. Doyle, B. Ladoux, and J.-L. Viovy, Physical Review Letters 84, 4769 (2000).

[46] J. Radler and E. Sackmann, Journal de Physique II France 3, 727 (1993).

[47] J.-B. Fournier and C. Barbetta, Phys. Rev. Lett. 100, 078103 (2008)

[48] M. Breidenich, R. R. Netz, and R. Lipowsky, EPL (Europhysics Letters) 49, 431 (2000).

[49] T. Bickel, C. M. Marques, and C. Jeppesen, Phys. Rev. E 62, 1124 (2000).

[50] M. Abramowitz and I. A. Stegun, Handbook of Mathematical Functions (Dover, 1970). 


\title{
Supplemental Material on \\ Lipid bilayer adhesion on sparse DNA carpets: theoretical analysis of membrane deformations induced by single end-grafted polymers.
}

\author{
F. Thalmann*, V. Billot, and C. M. Marques \\ Institut Charles Sadron, Université de Strasbourg, CNRS UPR 22, \\ 23 rue du Loess, F-67037 Strasbourg Cedex, France
}

(Dated: April 20, 2011)

We provide as Auxiliary Material a selection of deformation profiles obtained with the numerical procedure introduced in the Section IV of the main article. We also provide correspondance Tables relating the fixed adhesion $w$ situation and the fixed radius $L$ situation.

\section{SELECTION OF SELF-CONSISTENT QUANTITATIVE PROFILES}
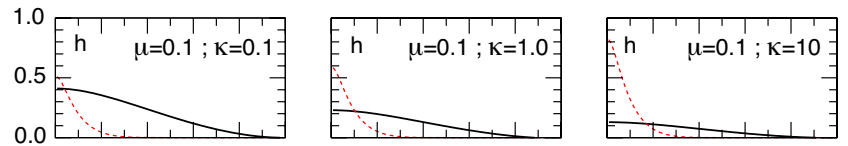

$1.0=h \quad \mu=0.2 ; \kappa=0.1=$
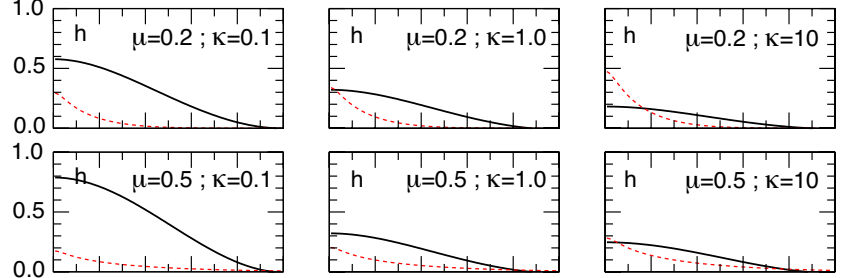

1.0
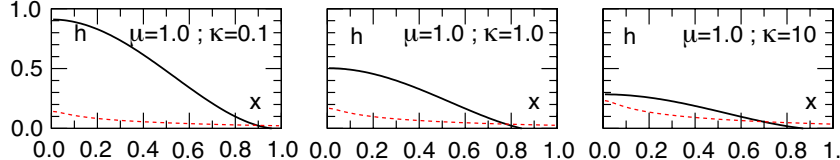

0.00 .20 .40 .60 .81 .00 .00 .20 .40 .60 .810

FIG. 1. Self-consistent solution for $\nu=0.1, L=1.0, T=1.0$ : from top to bottom and from left to right, $\kappa=0.1, \gamma=10^{-3}$, $R_{g}=0.1,0.2,0.5,1.0 ; \kappa=1, \gamma=10^{-2}, R_{g}=0.1,0.2,0.5,1.0 ;$ $\kappa=10, \gamma=0.1, R_{g}=0.1,0.2,0.5,1.0$. The pressure is shown in the same arbitrary units for all plots, as dashed lines.

The deformation profiles depend on three independant dimensionless combination of parameters. These parameters, introduced in Appendix $\mathrm{C}$ and $\mathrm{D}$, are $\nu=L / \xi$, $\mu=R_{g} / \xi$ and $\bar{\kappa}=\beta \kappa$, where $\xi=\sqrt{\kappa / \gamma}$ is the length scale where tension and curvature contribution match. $L$ serves as the unit of length and $k_{B} T$ as the unit of energy. Due to the large number of possible cases, we provide only a sample of the possible self-consistent profiles.

Figures (1),(2) and (3) present mosaic-like sets of profiles obtained for $\nu=0.1$ (curvature dominated case), $\nu=1$ (curvature and tension of same order of magnitude) and $\nu=10$ (tension dominated case, see caption for precise values). This total of 36 pictures correspond to a sampling of the three-dimensional parameter space $(\nu, \mu, \bar{\kappa})$ associated to the present problem.

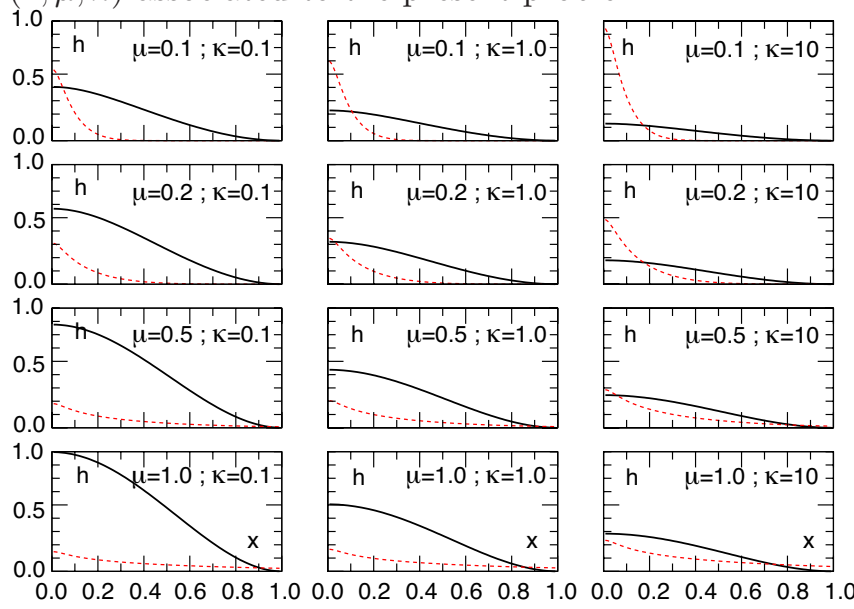

FIG. 2. Self-consistent solution for $\nu=1.0, L=1.0, T=1.0$ : from top to bottom and from left to right, $\kappa=0.1, \gamma=0.1$, $R_{g}=0.1,0.2,0.5,1.0 ; \kappa=1.0, \gamma=1.0, R_{g}=0.1,0.2,0.5,1.0$; $\kappa=10, \gamma=10, R_{g}=0.1,0.2,0.5,1.0$. The pressure is shown in the same arbitrary units for all plots, as dashed lines.

\section{PROFILES WITH FIXED ADHESION $w$}

When the adhesion parameter $w$ is fixed, one must determine the corresponding patch radius $L$. This amounts to solving a non-linear relation $w(L)$ for $L$. Table I provides a correspondence between the dimensionless parameters $\bar{\kappa}=\kappa / T, \bar{\gamma}=\gamma R_{g}^{2} / T$ and $\bar{w}=w R_{g}^{2}$ and the a priori unknown ratio $L / R_{g}$. Parameters $\mu$ and $\nu$ are also provided for completeness. Once the correspondence between $w$ and $L$ has been established, it is possible to refer to Figs (1), (2) and (3) in order to find out the actual profile. Table II gives the aspect ratio and $D / L$ and the adhesion parameter $w L^{2} / T$ in units $L, T$ corresponding to each triplet of parameters $(\bar{\kappa}, \nu, \mu)$. 

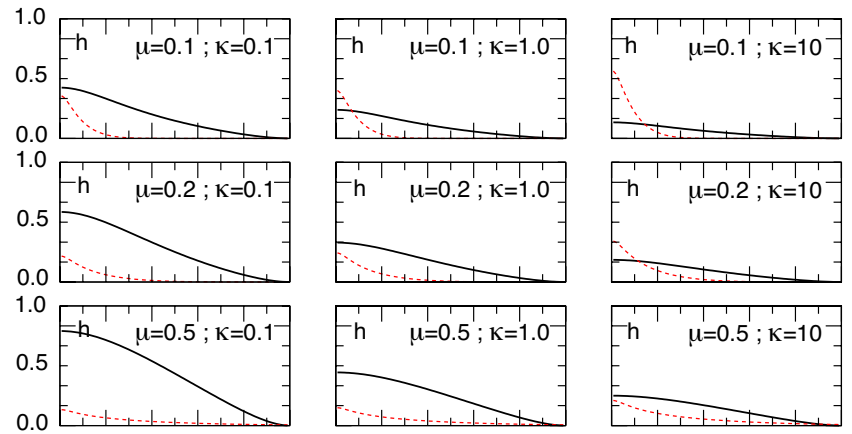

1.0

0.5
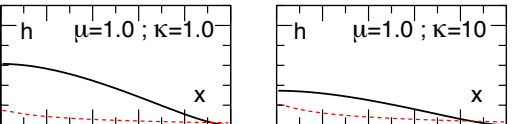

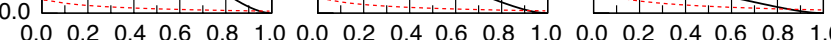

FIG. 3. Self-consistent solution for $\nu=10 ., L=1.0, T=$ 1.0: from top to bottom and from left to right, $\kappa=0.1$, $\gamma=10 ., R_{g}=0.1,0.2,0.5,1.0 ; \kappa=1.0, \gamma=100 ., R_{g}=$ $0.1,0.2,0.5,1.0 ; \kappa=10, \gamma=1000, R_{g}=0.1,0.2,0.5,1.0$. The pressure is shown in the same arbitrary units for all plots, as dashed lines.

\begin{tabular}{|c|c|c|c|c|c|c|}
\hline \multicolumn{1}{c}{$\bar{\gamma}$} & $\bar{\gamma}$ & $\bar{w}$ & $L / R_{g}$ & $D / R_{g}$ & $\mu=R_{g} / L$ & $\nu$ \\
\hline \hline 0.10 & 0.10 & 10.02 & 0.50 & 0.498 & 2.00 & 0.50 \\
0.10 & 0.10 & 0.997 & 1.27 & 1.082 & 0.79 & 1.27 \\
0.10 & 0.10 & 0.100 & 2.80 & 1.82 & 0.36 & 2.80 \\
\hline 0.10 & 1.00 & 10.09 & 0.505 & 0.484 & 1.98 & 1.60 \\
0.10 & 1.00 & 0.999 & 1.337 & 0.948 & 0.75 & 4.22 \\
0.10 & 1.00 & 0.101 & 3.030 & 1.313 & 0.33 & 9.58 \\
\hline 0.10 & 10.0 & 9.999 & 0.587 & 0.425 & 1.70 & 5.87 \\
0.10 & 10.0 & 0.9996 & 1.79 & 0.673 & 0.56 & 17.9 \\
0.10 & 10.0 & 0.1004 & 4.03 & 0.818 & 0.25 & 40.3 \\
\hline 1.00 & 0.10 & 9.91 & 0.807 & 0.429 & 1.24 & 0.255 \\
1.00 & 0.10 & 0.996 & 1.93 & 0.863 & 0.52 & 0.610 \\
1.00 & 0.10 & 0.1007 & 4.02 & 1.410 & 0.25 & 0.271 \\
\hline 1.00 & 1.00 & 10.02 & 0.813 & 0.425 & 1.23 & 0.813 \\
1.00 & 1.00 & 1.007 & 1.93 & 0.820 & 0.518 & 1.93 \\
1.00 & 1.00 & 0.1006 & 3.96 & 1.199 & 0.253 & 3.96 \\
\hline 1.00 & 10.0 & 10.02 & 0.837 & 0.400 & 1.20 & 2.65 \\
1.00 & 10.0 & 1.003 & 2.078 & 0.653 & 0.48 & 6.57 \\
1.00 & 10.0 & 0.1006 & 4.37 & 0.805 & 0.23 & 13.8 \\
\hline 10.0 & 0.10 & - & - & - & & \\
10.0 & 0.10 & 0.998 & 2.825 & 0.635 & 0.35 & 0.28 \\
10.0 & 0.10 & 0.1004 & 5.76 & 1.006 & 0.18 & 1.80 \\
\hline 10.0 & 1.00 & 10.03 & 1.272 & 0.351 & 0.786 & 0.402 \\
10.0 & 1.00 & 0.9980 & 2.825 & 0.635 & 0.354 & 0.893 \\
10.0 & 1.00 & 0.1003 & 5.68 & 0.956 & 0.176 & 1.796 \\
\hline 10.0 & 10.0 & 10.01 & 1.278 & 0.344 & 0.78 & 1.28 \\
10.0 & 10.0 & 0.9999 & 2.818 & 0.577 & 0.36 & 2.82 \\
10.0 & 10.0 & 0.1003 & 5.51 & 0.752 & 0.18 & 5.51 \\
\hline \hline & & & & & & \\
\hline \hline
\end{tabular}

TABLE I. This table lists the values of $L$ associated with prescribed values of adhesion $\bar{w}$. 


\begin{tabular}{|c|c|c|c|c|}
\hline \hline $\bar{\kappa}$ & $\nu$ & $\mu$ & $D / L$ & $w L^{2} / T$ \\
\hline \hline 0.10 & 0.10 & 0.10 & 0.3807 & $2.419 \times 10^{-3}$ \\
0.10 & 0.10 & 0.20 & 0.5255 & $2.654 \times 10^{-2}$ \\
0.10 & 0.10 & 0.50 & 0.6657 & 0.7703 \\
0.10 & 0.10 & 1.00 & 0.7122 & 8.057 \\
\hline 1.00 & 0.10 & 0.10 & 0.2646 & $2.190 \times 10^{-3}$ \\
1.00 & 0.10 & 0.20 & 0.3791 & $1.924 \times 10^{-2}$ \\
1.00 & 0.10 & 0.50 & 0.5515 & 0.2434 \\
1.00 & 0.10 & 1.00 & 0.6609 & 1.2865 \\
\hline 10.0 & 0.10 & 0.10 & 0.1585 & $4.734 \times 10^{-3}$ \\
10.0 & 0.10 & 0.20 & 0.2285 & $4.025 \times 10^{-2}$ \\
10.0 & 0.10 & 0.50 & 0.3371 & 0.4658 \\
10.0 & 0.10 & 1.00 & 0.4071 & 2.364 \\
\hline 0.10 & 1.00 & 0.10 & 0.4039 & $1.507 \times 10^{-3}$ \\
0.10 & 1.00 & 0.20 & 0.5672 & $1.504 \times 10^{-2}$ \\
0.10 & 1.00 & 0.50 & 0.7759 & 0.2791 \\
0.10 & 1.00 & 1.00 & 0.8965 & 1.8534 \\
\hline 1.00 & 1.00 & 0.10 & 0.2272 & $4.856 \times 10^{-3}$ \\
1.00 & 1.00 & 0.20 & 0.3190 & $4.843 \times 10^{-2}$ \\
1.00 & 1.00 & 0.50 & 0.4365 & 0.8987 \\
1.00 & 1.00 & 1.00 & 0.5043 & 5.9678 \\
\hline 10.0 & 1.00 & 0.10 & 0.1277 & $1.537 \times 10^{-2}$ \\
10.0 & 1.00 & 0.20 & 0.1794 & 0.1535 \\
10.0 & 1.00 & 0.50 & 0.2455 & 2.8484 \\
10.0 & 1.00 & 1.00 & 0.2836 & 18.913 \\
\hline 0.10 & 10.0 & 0.10 & 0.2543 & $1.264 \times 10^{-3}$ \\
0.10 & 10.0 & 0.20 & 0.3511 & $1.573 \times 10^{-2}$ \\
0.10 & 10.0 & 0.50 & 0.4739 & 0.4481 \\
0.10 & 10.0 & 1.00 & 0.5459 & 3.5954 \\
\hline 1.00 & 10.0 & 0.10 & 0.1430 & $4.071 \times 10^{-3}$ \\
1.00 & 10.0 & 0.20 & 0.1975 & $5.066 \times 10^{-2}$ \\
1.00 & 10.0 & 0.50 & 0.2664 & 1.442 \\
1.00 & 10.0 & 1.00 & 0.3070 & 11.58 \\
\hline 10.0 & 10.0 & 0.10 & 0.0804 & $1.290 \times 10^{-2}$ \\
10.0 & 10.0 & 0.20 & 0.1111 & 0.1605 \\
10.0 & 10.0 & 0.50 & 0.1498 & 4.5675 \\
10.0 & 10.0 & 1.00 & 0.1726 & 36.651 \\
\hline \hline & & & & \\
\hline 10
\end{tabular}

TABLE II. This table lists the values of $\bar{w}$ associated with prescribed values of adhesion $L$. 